\begin{tabular}{|r|l|}
\hline \multicolumn{2}{|c|}{ Statistica Sinica Preprint No: SS-2021-0117 } \\
\hline Title & $\begin{array}{l}\text { Cointegration Rank Estimation for High-Dimensional } \\
\text { Time Series With Breaks }\end{array}$ \\
\hline Uanuscript ID & SS-2021-0117 \\
\hline URL & http://www.stat.sinica.edu.tw/statistica/ \\
\hline DOI & $10.5705 /$ ss.202021.0117 \\
\hline Complete List of Authors & $\begin{array}{l}\text { Ngai Hang Chan and } \\
\text { Rongmao Zhang }\end{array}$ \\
\hline Corresponding Author & Ngai Hang Chan \\
\hline E-mail & nhchan@sta.cuhk.edu.hk \\
\hline Notice: Accepted version subject to English editing.
\end{tabular}




\title{
Cointegration Rank Estimation for High-Dimensional Time Series with Breaks
}

\author{
Ngai Hang Chan and Rongmao Zhang *
}

The Chinese University of Hong Kong and Zhejiang University

Abstract:

An intuitive and simple-to-use procedure for estimating the cointegration rank of a high-dimensional time series system with possible breaks is proposed in this paper. Based on a similar idea to principal component analysis, the cointegration rank can be estimated by the number of the eigenvalues of a certain non-negative definite matrix. There are several advantages to the new method: (a) the dimension of the cointegrated time series is allowed to vary with the sample size; (b) it is model-free; and (c) it is simple to use and robust against possible breaks in trend. The cointegration rank can be estimated without the need for a priori testing and estimating of the break points. Asymptotic properties of the proposed methods are investigated when the dimension of the time series increases with the sample size, which offers a new alternative to deal with high-dimensional time series. Illustrations of simulations are also reported.

Key words and phrases: Cointegration, eigenanalysis, high-dimensional time series, nonstationary processes, structural break.

\footnotetext{
*Corresponding author: Rongmao Zhang, rmzhang@zju.edu.cn
} 


\section{Introduction}

Cointegration was introduced to visualize the long-run equilibrium between several nonstationary economic series. During the past thirty years, cointegration analysis has attracted increasing attention from both theoretical and empirical researchers in economics and statistics alike. An excellent survey on the early developments in cointegration can be found in Johansen (1995).

Engle and Granger (1987) derived a representation for cointegration in the form of an error correction model (ECM), which reflects the correction of the long-run relationship with short-run dynamics. One of the remarkable features of ECM is that it clearly identifies the gain in prediction achieved using the cointegrated variables rather than the standard ARIMA approach. However, the associated inference method, now known as the Engle-Granger method, is designed for bivariate series only. The likelihood inference-based ECM is systematically developed in Johansen (1995). This likelihood approach is regarded as the most effective and efficient inference method for ECM. It provides the maximum likelihood estimator for cointegration spaces and likelihood ratio tests for cointegration ranks. However, the likelihood method is complex, requiring a correct specification of a finite order of the vector autoregression (VAR) for the short-run dynamic effec- 
t. In many applications, using different orders for the VAR would result in different conclusions on the cointegration. If the VAR order is underspecified or if the process lies outside the VAR class, then optimal inference on the unknown cointegration relations will lose validity, Hualde and Robinson (2010). Related methods, including the triangular system of Phillips (1991) and the frequency domain approach of Robinson and Yajima (2002), Müller and Watson (2013), also suffer similar shortcomings. Further, most of these methods focus mainly on fixed-dimensional cointegrated time series and they become impractical when dealing with high-dimensional series, see Ho and Sorensen (1996) and Gonzalo and Pitarakis (1999) for related discussions.

With the advancement of data acquisition technology, high-dimensional time series data are often encountered in finance and economics, e.g., highrisk assets in a large portfolio, or a large panel data in economics. A natural question is that if there exists a long-run equilibrium among the high-dimensional nonstationary economic series, then how can a cointegration relationship be detected? Recently, Onatski and Wang (2018) derived the asymptotic theory for high-dimensional setting, and Zhang, Robinson and Yao (ZRY, 2019) proposed a method for estimating the cointegration rank based on the eigenvectors of a certain positive definite ma- 
trix consisting of the sample covariance of the observed series $\left\{\mathbf{y}_{t}\right\}$, given by $\widehat{\mathbf{W}}=\sum_{j=0}^{j_{0}} \widehat{\Sigma}_{j} \widehat{\Sigma}_{j}^{\prime}$, where $\widehat{\mathbf{\Sigma}}_{j}=\frac{1}{n} \sum_{t=1}^{n-j}\left(\mathbf{y}_{t+j}-\overline{\mathbf{y}}\right)\left(\mathbf{y}_{t}-\overline{\mathbf{y}}\right)^{\prime}$ and $\overline{\mathbf{y}}=\frac{1}{n} \sum_{t=1}^{n} \mathbf{y}_{t}$ are the sample autocovariance and sample mean of the observations $\left\{\mathbf{y}_{t}: t=1, \ldots, n\right\}$ respectively. The symbol $H^{\prime}$ denotes the transpose of the matrix/vector $H$. Because ZRY employed a strong approximation technique to establish the asymptotic properties, they have to assume that the dimension of the time series cannot exceed $O\left(n^{1 / 4}\right)$. Further, ZRY did not consider the case with structural breaks in the trend, which can induce an apparent unit root phenomenon, see Zivot and Andrews (1992), Perron (1997), Cavaliere and Georgiev (2007) and the references therein. It has been shown that an unaccounted trend break can lead to substantial over-size and power loss in many standard tests for cointegration, see Campos, Ericsson and Hendry (1996) and Harris, Leybourne and Taylor (2016). Compared to the univariate case, relatively less work has been conducted for cointegration with structural breaks in the trend. Sporadic examples are given in Johansen, Mosconi and Nielsen (2000), Saikkonen and Lütkepohl (2000) and Trenkler, Saikkonen and Lütkepohl (2007). As pointed out by Harris, Leybourne and Taylor (2016), all these studies assume both a break exists and the lag order of the VAR model is known a priori. To overcome these difficulties, Harris, Leybourne and Taylor (2016) applied Johansen's 
likelihood ratio to test for the cointegration rank. To determine the cointegration rank, they further required a test at each step (an estimate for the break point and an order selection by Schwarz information criterion (SC) to be specific), which becomes difficult to implement for high-dimensional series.

The purpose of this paper is to propose a simple-to-use and robust to possible breaks approach to identify the cointegration rank in a highdimensional time series setting. To a certain extent, the proposed procedure is a reminiscence of principal component analysis. In classical principal component analysis, one looks for linear combinations of factors that give rise to large variation, which can be found by eigenvectors corresponding to large eigenvalues of the sample covariance matrix (see Anderson (1984)). Here, we search for linear combinations of integrated series that become stationary (cointegrated). Because eigenvalues of the long-run sample covariance matrix (see $\widehat{\mathbf{W}}_{x}$ below) of the difference of the stationary components are of smaller order than that of the nonstationary components, we then identify the cointegration space by means of the eigenvectors corresponding to small eigenvalues of the long-run sample covariance matrix of the time series. A similar idea was considered in Phillips and Ouliaris (1988), Harris (1997) and Robinson and Yajima (2002) for fixed dimensional cases, where 
they used the original series to recover the cointegration space.

Specifically, consider differencing the original series $\left\{\mathbf{y}_{t}\right\}$ to eliminate possible breaks. Note that for a $p$-dimensional observed series $\mathbf{y}_{t}$ with a linear trend, the differenced series $\nabla \mathbf{y}_{t}=\mathbf{y}_{t}-\mathbf{y}_{t-1}$ would only have a break at the change point. To elucidate this idea, consider a simple example with one possible break in level (cases with multiple breaks can be handled similarly). Let $\mathbf{y}_{t}=\mathbf{c}_{1}+\mathbf{c}_{2} I\left(t \geq t_{0}\right)+\mathbf{d} t+\boldsymbol{X}_{t}$, where $\mathbf{c}_{i}, i=1,2$ and $\mathbf{d}$, are $p$-dimensional constant vectors, and $\boldsymbol{X}_{t}$ is a $p$-dimensional cointegrated process with no trend. Then

$$
\nabla \mathbf{y}_{t}=\mathbf{d}+\mathbf{c}_{2} I\left(t=t_{0}\right)+\nabla \boldsymbol{X}_{t}
$$

The trend of $\nabla \mathbf{y}_{t}$ reduces to a constant except at the change point $t=t_{0}$. We can therefore remove the trend by considering $\nabla \mathbf{y}_{t}-\nabla \overline{\mathbf{y}}$, to recover the cointegration relationship, where $\nabla \overline{\mathbf{y}}=\frac{1}{n} \sum_{t=1}^{n} \nabla \mathbf{y}_{t}$. Because the number of break points is small, using $\nabla \mathbf{y}_{t}-\nabla \overline{\mathbf{y}}$ to estimate the cointegration rank will have little effect on the performance. The distinct advantage of this method is that it avoids estimating and testing for the trend with possible breaks.

Furthermore, we propose to recover the cointegration space based on a weighted matrix function of the sample autocovariance of the differenced process. Note that if $\boldsymbol{X}_{t}$ is cointegrated with rank $r$, then there exist a 
$p \times p$ matrix $\mathbf{A}$ and a $p$-dimensional series $\mathbf{z}_{t}$ with $r$ stationary components and $p-r$ nonstationary components such that

$$
\boldsymbol{X}_{t}=\mathrm{Az}_{t}
$$

(see Zhang, Robinson and Yao (2019)). It follows from (1.1) that the differenced process $\left\{\nabla \boldsymbol{X}_{t}\right\}$ satisfies

$$
\nabla \boldsymbol{X}_{t}=\mathbf{A} \nabla \mathbf{z}_{t}
$$

We adopt a similar approach of ZRY to estimate the cointegration space, but based on a different sample covariance matrix. Let $\widehat{\Sigma}_{j, x}$ be the sample autocovariances between $\nabla \boldsymbol{X}_{t}$ and $\nabla \boldsymbol{X}_{t+j}, j \geq 0$. That is,

$$
\widehat{\boldsymbol{\Sigma}}_{j, x}=\frac{1}{n-j} \sum_{i=1}^{n-j}\left(\nabla \boldsymbol{X}_{t+j}-\nabla \overline{\boldsymbol{X}}\right)\left(\nabla \boldsymbol{X}_{t}-\nabla \overline{\boldsymbol{X}}\right)^{\prime}
$$

and

$$
\widehat{\mathbf{W}}_{x}=\sum_{j=0}^{M} w(j)\left(\widehat{\Sigma}_{j, x}+\widehat{\mathbf{\Sigma}}_{j, x}^{\prime}\right),
$$

with $w(0)=1 / 2$ and $w(j)=(1-j /(M+1))$ for $j \geq 1$.

Then, $\widehat{\mathbf{W}}_{x}$ is a symmetric and positive definite matrix, see Newey and West (1987). Because the convergent rates of the eigenvalues of $\widehat{\mathbf{W}}_{x}$ are different for cointegrated and non-cointegrated space (see (2.3) and (2.4) below), we can recover the cointegration space by the eigenvectors corresponding to the $r$ smallest eigenvalues of $\widehat{\mathbf{W}}_{x}$. We also propose two methods 
to estimate the cointegration rank $r$ : one based on an information criterion and the other one based on the ratio of the eigenvalues of $\widehat{\mathbf{W}}_{x}$. Moreover, we establish the consistency of the estimated cointegration space and rank for both fixed and diverging $p$.

Compared to other procedures based on Engle and Granger's (1987) ECM, the proposed method has several advantages. First, it avoids misspecification or lag order selection for the VAR model of the short-run effect. Second, unlike other results, our method works regardless of the existence of breaks in the trend. Third, we allow the dimension $p$ to diverge with $n$ with a rate faster than that of ZRY.

Throughout the paper, $\|\cdot\|$ denotes the spectral norm of a matrix. In particular, for a matrix $\mathbf{H},\|\mathbf{H}\|=\sqrt{\lambda_{\max }\left(\mathbf{H}^{\prime} \mathbf{H}\right)}$, where $\lambda_{\max }(\cdot)$ denotes the largest eigenvalue of a matrix. The rest of the paper is organized as follows. The proposed methodology is presented in Section 2. Asymptotic theory is developed in Section 3. Numerical studies are reported in Section 4, and Section 5 is the conclusion. Technical proofs are relegated to the supplementary material.

\section{Methods}

\subsection{Setting}


We call a vector process $\mathbf{u}_{t}$ weakly stationary if (i) $E \mathbf{u}_{t}$ is a constant vector independent of $t$, and (ii) $E\left\|\mathbf{u}_{t}\right\|^{2}<\infty$, and $\operatorname{Cov}\left(\mathbf{u}_{t}, \mathbf{u}_{t+s}\right)$ depends on $s$ only for any integers $t, s$, where $\|\cdot\|$ denotes the Euclidean norm. Denote by $\nabla$ the difference operator, i.e. $\nabla \mathbf{u}_{t}=\mathbf{u}_{t}-\mathbf{u}_{t-1}$. Further, if $\mathbf{u}_{t}$ has a spectral density matrix that is finite and definitely positive at zero frequency, then we say $\mathbf{u}_{t}$ is an $I(0)$ process. An example of an $I(0)$ process is a stationary and invertible vector ARMA. We say $\mathbf{x}_{t}$ is a $p$-dimensional integrated process of order 1 (i.e., I(1)) if all its components follow an unitroot process, i.e., $\nabla \mathbf{x}_{t}=\mathbf{u}_{t}$. Now assume $\mathbf{y}_{t}$ is a $p$-dimensional observable time series with a linear trend and admits the form: $\mathbf{y}_{0}=0$ and

$$
\mathbf{y}_{t}=\sum_{i=1}^{m+1} \mathbf{a}_{i} I\left(t_{i-1}<t \leq t_{i}\right)+\mathbf{b} t+\mathbf{x}_{t}, \quad \text { and } \quad \mathbf{x}_{t}=\mathbf{A} \mathbf{z}_{t}
$$

where $0=t_{0}<t_{1}<\cdots<t_{m+1}=n, \mathbf{b}, \mathbf{a}_{i}$ s are $p$-dimensional constant vectors, $\mathbf{A}$ is an unknown and invertible constant matrix, $\mathbf{x}_{t}$ is a latent p-dimensional $I(1)$ process, $\mathbf{z}_{t}=\left(\mathbf{z}_{t 1}^{\prime}, \mathbf{z}_{t 2}^{\prime}\right)^{\prime}$ is a $p$-dimensional process, $\mathbf{z}_{t 2}$ is an $r$-dimensional $I(0)$ process, and $\mathbf{z}_{t 1}$ is a $(p-r)$-dimensional $I(1)$ process. Furthermore, no linear combination of $\mathbf{z}_{t 1}$ is $I(0)$ because such a stationary variable can be subsumed into $\mathbf{z}_{t 2}$. Each component of $\mathbf{z}_{t 2}$ is a cointegrating error of $\mathbf{x}_{t}$ and $r \geq 0$ is the cointegration rank. In the event that there exists no cointegration among the components of $\mathbf{x}_{t}, r=0$. When $\mathbf{x}_{t}$ itself is an $I(0)$ process, then $r=p$. For many economic and financial applications, 
there exists a small number of cointegrated variables, i.e. $r \geq 1$ is a small integer. Expression (2.1) was considered in ZRY for the case without a trend, i.e., all the coefficients $\mathbf{a}_{i} \mathrm{~s}$ and $\mathbf{b}$ are zero.

The pair $\left(\mathbf{A}, \mathbf{z}_{t}\right)$ in (2.1) is not uniquely defined, as it can be replaced by $\left(\mathbf{A H}^{-1}, \mathbf{H} \mathbf{z}_{t}\right)$ for any invertible $\mathbf{H}$ of the form

$$
\left(\begin{array}{cc}
\mathbf{H}_{11} & \mathbf{H}_{12} \\
\mathbf{0} & \mathbf{H}_{22}
\end{array}\right),
$$

where $\mathbf{H}_{11}, \mathbf{H}_{22}$ are square matrices of size $(p-r), r$ respectively, and $\mathbf{0}$ denotes a matrix with all entries equal to 0 . Therefore, there is no loss of generality in assuming $\mathbf{A}$ to be orthogonal, because any non-orthogonal $\mathbf{A}$ admits the decomposition $\mathbf{A}=\mathbf{Q} \mathbf{U}$, where $\mathbf{Q}$ is orthogonal and $\mathbf{U}$ is upper-triangular, and we may then replace $\left(\mathbf{A}, \mathbf{z}_{t}\right)$ in $(2.1)$ by $\left(\mathbf{Q}, \mathbf{U} \mathbf{z}_{t}\right)$. In the sequel, we always assume that $\mathbf{A}$ in (2.1) is orthogonal, i.e., $\mathbf{A}^{\prime} \mathbf{A}=\mathbf{I}_{p}$, where $\mathbf{I}_{p}$ denotes the $p \times p$ identity matrix. Write

$$
\mathbf{A}=\left(\mathbf{A}_{1}, \mathbf{A}_{2}\right)
$$

where $\mathbf{A}_{1}$ and $\mathbf{A}_{2}$ are respectively, $p \times(p-r)$ and $p \times r$ matrices. As $\mathbf{z}_{t 2}=\mathbf{A}_{2}^{\prime} \mathbf{x}_{t}$, the linear space spanned by the columns of $\mathbf{A}_{2}$, denoted by $\mathcal{M}\left(\mathbf{A}_{2}\right)$, is called the cointegration space. In fact this cointegration space is uniquely defined by (2.1). 


\subsection{Estimation}

The goal is to determine the cointegration rank $r$ in (2.1) and to identify $\mathbf{A}_{2}$, or more precisely $\mathcal{M}\left(\mathbf{A}_{2}\right)$. Then $\mathcal{M}\left(\mathbf{A}_{1}\right)$ is the orthogonal complement of $\mathcal{M}\left(\mathbf{A}_{2}\right)$, and $\mathbf{z}_{i t}=\mathbf{A}_{i}^{\prime} \mathbf{x}_{t}$ for $i=1,2$. Our estimation method is motivated by the following observation. To remove the trend effect, we consider the first difference of $\mathbf{y}_{t}$. From (2.1), it follows that

$$
\nabla \mathbf{y}_{t}=\mathbf{b}+\sum_{i=1}^{m}\left(\mathbf{a}_{i+1}-\mathbf{a}_{i}\right) I\left(t=t_{i}+1\right)+\nabla \mathbf{x}_{t} .
$$

From (2.2), we know that the trend of $\nabla \mathbf{y}_{t}$ only changes at the break points $t_{i}+1$ for $i=1,2, \ldots, m$. If we ignore these break points and consider $\nabla \mathbf{y}_{t}$ as a process with a constant mean, then it can be detrended by subtracting the sample mean, $\nabla \overline{\mathbf{y}}=\frac{1}{n} \sum_{t=1}^{n} \nabla \mathbf{y}_{t}$. That is, consider $\nabla \widehat{\mathbf{x}}_{t}=\nabla \mathbf{y}_{t}-\nabla \overline{\mathbf{y}}$ and recover the cointegration space based on the sample covariance of $\nabla \widehat{\mathbf{x}}_{t}$. In particular, for $j \geq 0$, we define

$$
\widehat{\mathbf{\Sigma}}_{j}=\frac{1}{n-j} \sum_{t=1}^{n-j} \nabla \widehat{\mathbf{x}}_{t+j} \nabla \widehat{\mathbf{x}}_{t}^{\prime}
$$

and define $\mathbf{W}$ as in (1.3), i.e., let $w(0)=1 / 2$ and $w(j)=(1-j /(M+1))$ for $j \geq 1$ and

$$
\widehat{\mathbf{W}}=\sum_{j=0}^{M} w(j)\left(\widehat{\boldsymbol{\Sigma}}_{j}+\widehat{\boldsymbol{\Sigma}}_{j}^{\prime}\right) .
$$

Due to possible breaks, $\left\{\nabla \widehat{\mathbf{x}}_{t}\right\}$ is not stationary and $\widehat{\mathbf{W}}$ is not an exact estimator of the spectral density of $\nabla \mathbf{x}_{t}$ at frequency zero. But $\left\{\nabla \widehat{\mathbf{x}}_{t}\right\}$ is 
asymptotically stationary and consequently $\widehat{\mathbf{W}}$ can still estimate the spectral density consistently at frequency zero. It can be shown that under some mild conditions, for any $\mathbf{c} \in \mathcal{M}\left(\mathbf{A}_{2}\right)$,

$$
\mathbf{c}^{\prime} \widehat{\mathbf{W}} \mathbf{c}=O_{p}\left(1 / M+B_{n M p}\right)=o_{p}(1)
$$

as $n, M \rightarrow \infty$, where $B_{n M p}$ is given in (3.3) below. However for any $\mathbf{c} \notin$ $\mathcal{M}\left(\mathrm{A}_{2}\right)$

$$
\mathbf{c}^{\prime} \widehat{\mathbf{W}} \mathbf{c}=O_{e}(1)
$$

where $U=O_{e}(V)$ indicates that $P(0<|U / V|<\infty) \rightarrow 1$. Intuitively the $r$ directions of the cointegration space $\mathcal{M}\left(\mathbf{A}_{2}\right)$ make $\left|\mathbf{c}^{\prime} \widehat{\mathbf{W}} \mathbf{c}\right|$ as small as possible. Consequently, $\mathcal{M}\left(\mathbf{A}_{2}\right)$ can be estimated by the linear space spanned by the $r$ eigenvectors of $\widehat{\mathbf{W}}$ corresponding to the $r$ smallest eigenvalues, and $\mathcal{M}\left(\mathbf{A}_{1}\right)$ can be estimated by that spanned by the $(p-r)$ eigenvectors of $\widehat{\mathbf{W}}$ corresponding to the $(p-r)$ largest eigenvalues.

Let $\left(\widehat{\gamma}_{1}, \ldots, \widehat{\gamma}_{p}\right)$ be the orthogonal eigenvectors of $\widehat{\mathbf{W}}$ that correspond to the eigenvalues arranged in descending order. Define

$$
\widehat{\mathbf{A}}=\left(\widehat{\mathbf{A}}_{1}, \widehat{\mathbf{A}}_{2}\right), \widehat{\mathbf{A}}_{1}=\left(\widehat{\gamma}_{1}, \ldots, \widehat{\gamma}_{p-r}\right), \widehat{\mathbf{A}}_{2}=\left(\widehat{\gamma}_{p-r+1}, \ldots, \widehat{\gamma}_{p}\right)
$$

Then $\mathcal{M}\left(\widehat{\mathbf{A}}_{1}\right)$ and $\mathcal{M}\left(\widehat{\mathbf{A}}_{2}\right)$, the linear spaces spanned by the eigenvectors of $\widehat{\mathbf{W}}$, are consistent estimators for $\mathcal{M}\left(\mathbf{A}_{1}\right)$ and $\mathcal{M}\left(\mathbf{A}_{2}\right)$ respectively; see Theorem 1 below. 
The idea of using an eigenanalysis based on sample autocovariance matrices has been used for factor modeling for dimension reduction (Lam and Yao (2012), and references therein), and for identifying cointegration space (ZRY). These papers are based on a quadratic form of the sample autocovariance of the observed series $\mathbf{y}_{t}$, which requires estimating the trend and testing for possible break points. By virtue of (2.2) to (2.5), these difficulties can be circumvented by means of $\nabla \mathbf{y}_{t}$.

\subsection{Determining Cointegration Ranks}

We introduce two criteria to estimate the cointegration rank $r$ based on the estimated eigenvalues of $\widehat{\mathbf{W}}$. Let $\widehat{\lambda}_{1} \geq \cdots \geq \widehat{\lambda}_{p} \geq 0$ be the eigenvalues of $\widehat{\mathbf{W}}$ and $\nu_{n}=M^{-1}+B_{n M p}$. By virtue of (2.3) and (2.4), $\widehat{\lambda}_{i}$ converges to some positive constant for all $1 \leq i \leq p-r$, and $\widehat{\lambda}_{i}=O_{p}\left(\nu_{n}\right)$ for all $p-r<i \leq p$. Hence for $1 \leq r<p, \widehat{\lambda}_{i} / \nu_{n}^{1 / 2} \rightarrow \infty$ in probability for all $1 \leq i \leq p-r$. For $p-r<i \leq p, \widehat{\lambda}_{i} / \nu_{n}^{1 / 2}=o_{p}(1)$. This leads to estimating $r$ by

$$
\widehat{r}=\max \left\{j: \widehat{\lambda}_{p+1-j} / \nu_{n}^{1 / 2} \leq 1,1 \leq j \leq p\right\}
$$

which can be seen as a revised ratio estimate of Lam and Yao (2012). However, Lam and Yao's estimator is not consistent, but $\widehat{r}$ is; see Theorem 2 below. 
Alternatively, we may define a so-called information criterion as follows

$$
I C(l)=\sum_{j=1}^{l} \widehat{\lambda}_{p+1-j}+(p-l) \omega_{n},
$$

where the first term is used to fit the cointegrated components and the second term is the penalty for lack of fit and $\omega_{n} \rightarrow 0$. Then $r$ can be estimated by

$$
\widetilde{r}=\arg \min _{1 \leq l \leq p} I C(l) .
$$

Note that when $\omega_{n}=\nu_{n}^{1 / 2}, \widetilde{r}=\widehat{r}$. The consistency of $\widetilde{r}$ is established in Theorem 2 below.

\section{Asymptotic Properties}

In this section, we investigate the asymptotic properties of the proposed estimators. First, we show that with $r$ given, the linear space $\mathcal{M}\left(\widehat{\mathbf{A}}_{2}\right)$ is a consistent estimate for the cointegration space $\mathcal{M}\left(\mathbf{A}_{2}\right)$. We measure the distance between the two spaces by

$$
D\left(\mathcal{M}\left(\widehat{\mathbf{A}}_{2}\right), \mathcal{M}\left(\mathbf{A}_{2}\right)\right)=\sqrt{1-\frac{1}{r} \operatorname{tr}\left(\widehat{\mathbf{A}}_{2} \widehat{\mathbf{A}}_{2}^{\prime} \mathbf{A}_{2} \mathbf{A}_{2}^{\prime}\right)} .
$$

Then $D\left(\mathcal{M}\left(\widehat{\mathbf{A}}_{2}\right), \mathcal{M}\left(\mathbf{A}_{2}\right)\right) \in[0,1]$, being 0 if and only if $\mathcal{M}\left(\widehat{\mathbf{A}}_{2}\right)=\mathcal{M}\left(\mathbf{A}_{2}\right)$, and 1 if and only if $\mathcal{M}\left(\widehat{\mathbf{A}}_{2}\right)$ and $\mathcal{M}\left(\mathbf{A}_{2}\right)$ are orthogonal. Furthermore, we show that both estimators $\widehat{r}$ and $\widetilde{r}$, defined in (2.6) and (2.7) respectively, are consistent. We consider two asymptotic regimes: (i) $p$ is fixed while $n \rightarrow \infty$, and (ii) $p \rightarrow \infty$ as $n \rightarrow \infty$. 
Put $\mathbf{z}_{t 1}=\left(z_{t, 1}, \ldots, z_{t, p-r}\right)^{\prime}$ and $\mathbf{z}_{t 2}=\left(z_{t, p-r+1}, \ldots, z_{t, p}\right)^{\prime}$. Under (2.1), $z_{t, j}$ is $I(1)$ for $1 \leq j \leq p-r$ and $e_{t, j} \equiv \nabla z_{t, j}$ is weakly stationary, i.e., an $I(0)$ process. Write $\boldsymbol{e}_{t 1}=\left(e_{t, 1}, \ldots, e_{t, p-r}\right)^{\prime}$ and $\boldsymbol{e}_{t}=\left(\boldsymbol{e}_{t 1}^{\prime}, \mathbf{z}_{t 2}^{\prime}\right)^{\prime}$. We introduce the following regularity conditions.

\section{Regularity Conditions.}

(i) There exists a sequence of independent and identically distributed (i.i.d.) random vectors $\varepsilon_{t}=\left(\varepsilon_{t 1}, \varepsilon_{t 2}, \ldots, \varepsilon_{t l}\right)^{\prime}$ such that

$$
\boldsymbol{e}_{t}=\sum_{j=-\infty}^{t} \mathbf{C}_{t-j} \varepsilon_{j}
$$

where $\varepsilon_{t i}, 1 \leq i \leq l$ are independent random variables with mean zero and $\max _{1 \leq i \leq l} \mathrm{E}\left|\varepsilon_{t i}\right|^{4}<\infty$, and $\mathbf{C}_{j}, j \geq 0$ are $p \times l$ matrices satisfying

$$
\sum_{j=0}^{\infty}\left\|\mathbf{C}_{j}\right\|<\infty \text { and } \lambda_{\min }\left(\sum_{j=0}^{\infty} \mathbf{C}_{j} \mathbf{C}_{j}^{T}\right)>\delta
$$

for some $\delta>0$, herein, $\lambda_{\min }$ denotes the smallest eigenvalue of a matrix.

(ii) The bandwidth $M$ defined in (1.3) and the dimensions $p$ satisfy that $M \rightarrow \infty$ and

$$
B_{n M p}=\frac{M \sqrt{p} \sum_{i=1}^{m}\left\|\mathbf{a}_{i+1}-\mathbf{a}_{i}\right\|}{n}+\sqrt{\frac{M \min \left(p^{2}, l^{2}\right)}{n}} \rightarrow 0
$$

as $n \rightarrow \infty$. 
Remark 1. Model (3.2) includes a large class of linear processes, for example the vector ARMA process. The process when $l=p$ with fixed $p$, (3.2) has been studied by many authors (see for example Marinucci and Robinson (2000)). However, little is known about the large $p$ case. Recently, Chen and $\mathrm{Wu}(2019)$ considered the process (3.2) for the large $p$ case under the assumption that $\varepsilon_{t i}, t, i \in \mathcal{Z}$ are i.i.d. random variables and assume that $\sum_{j=0}^{\infty}\left\|\mathbf{C}_{j}\right\|_{F}<\infty$, where $\|\cdot\|_{F}$ denotes the Frobenius norm of a matrix. Note that $\sum_{j=0}^{\infty}\left\|\mathbf{C}_{j}\right\|<\infty$ is weaker than that of $\sum_{j=0}^{\infty}\left\|\mathbf{C}_{j}\right\|_{F}<\infty$ and is sufficient to ensure that $\sum_{k=0}^{\infty}\left\|\mathrm{E}\left(\boldsymbol{e}_{k} \boldsymbol{e}_{0}^{T}\right)\right\|<\infty$, which implies that the important measure of the stability (the spectral density) of $\boldsymbol{e}_{t}$ exists and is bounded and continuous (Basu and Michailidis (2015)). The assumption of independence of the elements of $\varepsilon_{t}$ is imposed for simplicity in presenting the proof. When $l \leq p$, the independent assumption can be replaced by $\mathrm{E}\left(\left\|\varepsilon_{t} \varepsilon_{t}^{T}\right\|^{2}\right)=O(l)$

Remark 2. When $\sum_{j=0}^{\infty}\left\|\mathbf{C}_{j}\right\|_{F}<\infty$, we can replace $B_{n M p}$ by

$$
B_{n M p}^{*}=\frac{M \sqrt{p} \sum_{i=1}^{m}\left\|\mathbf{a}_{i+1}-\mathbf{a}_{i}\right\|}{n}+\sqrt{\frac{M}{n}} \rightarrow 0 .
$$

Remark 3. It is easy to see that the $B_{n M p}$ in Condition 1(ii) satisfies $B_{n M p}=O\left(\sqrt{M p^{2} / n}\right)$. Further, when $l$ is finite, then Condition (ii) reduces to $M \sqrt{p} \sum_{i=1}^{m}\left\|\mathbf{a}_{i+1}-\mathbf{a}_{i}\right\| / n \rightarrow 0$, which depends on the jump size of the 
common breaks $\sum_{i=1}^{m}\left\|\mathbf{a}_{i+1}-\mathbf{a}_{i}\right\|$. If only a finite number of components of $\mathbf{y}_{t}$ have breaks in level at each break points, then $\sum_{i=1}^{m}\left\|\mathbf{a}_{i+1}-\mathbf{a}_{i}\right\|=$ $O(1)$ and Condition (ii) is equivalent to $M \sqrt{p} / n \rightarrow 0$. As a result, $p$ is allowed to be bigger than the sample size $n$ when $M=o\left(n^{1 / 2}\right)$. When $l \geq p$, because the number $m$ of break points is always finite, Condition (ii) reduces to $M p^{2} / n \rightarrow 0$. In all cases, under the forth moment condition and $M=o\left(n^{1 / 4}\right)$ as in Theorem 2 of Newey and West (1987), $p$ is allowed to be larger than $O\left(n^{3 / 8}\right)$, which is much larger than that of ZRY.

Theorem 1. Let $r$ be given. Under Condition 1, we have

$$
\|\widehat{\mathbf{A}}-\mathbf{A}\|=O_{p}\left(B_{n M p}\right) .
$$

For the eigenvalues $\widehat{\lambda}_{1} \geq \cdots \geq \lambda_{p}$ of $\widehat{\mathbf{W}}$, we have

$$
\lambda_{p-r}=O_{e}(1) \quad \text { and } \quad \lambda_{p-r+1}=O_{p}\left(1 / M+B_{n M p}\right) .
$$

For the cointegration space, we have

$$
D\left(\mathcal{M}\left(\widehat{\mathbf{A}}_{2}\right), \mathcal{M}\left(\mathbf{A}_{2}\right)\right)=O_{p}\left(B_{n M p}\right) .
$$

Theorem 2. Let $1 \leq r<p$ and Condition 1 hold.

(i) For $\widehat{r}$ defined in (2.6), $\lim _{n \rightarrow \infty} P(\widehat{r}=r)=1$.

(ii) For $\widetilde{r}$ defined in 2.7), $\lim _{n \rightarrow \infty} P(\widetilde{r}=r)=1$ provided $\omega_{n} \rightarrow 0, \omega_{n} / \nu_{n} \rightarrow$ $\infty$. 
Remark 4. Theorem 1 shows that as $B_{n M p} \rightarrow 0$ and $M \rightarrow \infty$, one can recover the cointegration space consistently without knowing a priori if breaks exist. Theorem 2 shows that when the cointegration rank $r$ is unknown, then one can estimate it consistently based on ratio or information criterion of eigenvalues of $\widehat{\mathbf{W}}$ without estimating and testing for the possible breaks. And the conclusions of Theorem 1 still hold by replacing $r$ with its estimator. Hence, the proposed method is much simpler than the others and is applicable to high-dimensional cointegrated series.

\section{Numerical Results}

We illustrate the proposed method with five simulated examples. Comparison with Johansen's (1991) likelihood method is also conducted in Example 1. Examples 2-4 illustrate the method in the presence of a break point, in which we also compare the proposed method with that based on unit-root test, like ACF (sample autocorrelation function) method of ZRY (see also Zhang and Chan (2018)) and the Phillips-Perron test (PP.test). Example 5 applies the method to the case with breaks in a linear trend. We also applied the method to the monthly exchange rates of the U.S. dollar versus the currencies of 19 OECD countries in Example 6. In view of Condition 1(ii), the dimension $p$ is usually no bigger than $n^{1 / 2}$. Therefore, 
we have only considered $p \leq 80$ in these examples, which is slightly bigger than the square root of the sample size $n=2500$.

Example 1. To facilitate the computation, in model (2.1), let $\mathbf{a}_{1}=\cdots=$ $\mathbf{a}_{m}$ be a $p$-dimensional vector with each component independently generated from $U(-1,1)$. Likewise, let $\mathbf{b}$ be a $p$-dimensional vector with each component independently generated from $U(1,2)$ and let $\mathbf{z}_{t 2}$ consist of $r$ stationary $\mathrm{AR}(1)$ processes with coefficients generated from $U(-0.5,0.5)$, and let the remaining $p-r$ components of $\mathbf{z}_{t 1}$ be $\operatorname{ARIMA}(1,1,1)$ models with $\mathrm{AR}$ and MA coefficients generated independently from $U(0,0.6)$ and $U(0,0.8)$ respectively. All innovations involved are independent $N(0,1)$ random variables. Such a setting ensures all regularity conditions are valid. Let $\mathbf{A}$ be an orthogonalized version of a matrix with elements generated independently from $U(-3,3)$. We estimate the cointegration rank $r$ by (2.7) with $\omega_{n}=\nu_{n}^{1 / 3}, \nu_{n}=M^{-1}+\bar{B}_{n M P}$ and $M=50$ in $\widehat{\mathbf{W}}$. For each setting, we replicate the exercise 500 times. We then estimate $\widehat{\mathbf{A}}$ by (2.5) . Because $\widetilde{r}$ or $\widehat{r}$ is not necessarily equal to $r$, we extend the definition of discrepancy measure (3.1) as follows:

$$
D_{1}^{*}\left(\mathcal{M}\left(\widehat{\mathbf{A}}_{2}\right), \mathcal{M}\left(\mathbf{A}_{2}\right)\right)=\left\{1-\frac{\operatorname{tr}\left(\widehat{\mathbf{A}}_{2} \widehat{\mathbf{A}}_{2}^{\prime} \mathbf{A}_{2} \mathbf{A}_{2}^{\prime}\right)}{\max (r, \widehat{r})}\right\}^{1 / 2} .
$$

When $\widehat{r}=r, D_{1}^{*}\left(\mathcal{M}\left(\widehat{\mathbf{A}}_{2}\right), \mathcal{M}\left(\mathbf{A}_{2}\right)\right)=D\left(\mathcal{M}\left(\widehat{\mathbf{A}}_{2}\right), \mathcal{M}\left(\mathbf{A}_{2}\right)\right)$ defined in (3.1) 
The relative frequencies (RF) for the occurrence of the event $\{\widehat{r}=r\}$ and the average value of $D_{1}=D_{1}^{*}\left(\mathcal{M}\left(\widehat{\mathbf{A}}_{2}\right), \mathcal{M}\left(\mathbf{A}_{2}\right)\right)$ for 500 replications are listed in Table 1 under the label IC.

Because cointegration rank is the number of the stationary components of $\mathbf{z}_{t}$, one can also estimate the rank by unit-root test (see Engle and Granger (1987)). We also include the results of the unit-root test estimation introduced by Zhang and Chan (2018) (see also ZRY) in Table 1 under the label ACF. In particular, let $\widehat{\mathbf{x}}_{t}=\mathbf{y}_{t}-\widehat{\mathbf{a}}-\widehat{\mathbf{b}} t$ and

$$
\widehat{\mathbf{z}}_{t}=\left(\widehat{z}_{t, 1}, \ldots, \widehat{z}_{t, p}\right)^{\prime}=\widehat{\mathbf{A}}^{\prime} \widehat{\mathbf{x}}_{t}
$$

where $(\widehat{\mathbf{a}}, \widehat{\mathbf{b}})$ is given by

$$
(\widehat{\mathbf{a}}, \widehat{\mathbf{b}})=\operatorname{argmin}_{(\mathbf{a}, \mathbf{b})} \sum_{t=1}^{n}\left(\mathbf{y}_{t}-\mathbf{a}-\mathbf{b} t\right)^{\prime}\left(\mathbf{y}_{t}-\mathbf{a}-\mathbf{b} t\right) .
$$

Note that the order of the components inversely reflects the closeness to stationarity of the component series, with the last component $\left\{\widehat{z}_{t, p}\right\}$ most likely being stationary, and the first component $\left\{\widehat{z}_{t, 1}\right\}$ most likely being an $I(1)$ process. Let $\widehat{\rho}_{i}(\cdot)$ be the ACF of $\widehat{z}_{t, i}$ given by

$$
\widehat{\rho}_{i}(k)=\widehat{\gamma}_{i}(k) / \widehat{\gamma}_{i}(0),
$$

where $\widehat{\gamma}_{i}(k)=\frac{1}{n} \sum_{t=1}^{n-k} \widehat{z}_{t+k, i} \widehat{z}_{t, i}, \quad i=1,2, \ldots, p$ and define

$$
T_{n, i}=\frac{n}{q(q+1)} \sum_{k=1}^{q}\left(\widehat{\rho}_{i}(k)-1\right) .
$$


We estimate the cointegration rank $r$ by

$$
\widehat{r}^{*}=\sum_{i=1}^{p} I\left\{T_{n, i}<-a_{n}\right\}
$$

where $a_{n} \rightarrow \infty$ and $R$ is a prescribed upper bound of $r$. In our numerical experiments, we set $a_{n}=(n / q)^{1 / 2}, q=\left[n^{1 / 4}\right]$.

To illustrate the performance of the proposed method, in Table 1 we also report the results of Johansen's likelihood estimation with cointegration rank $r$ estimated by the trace test; see Johansen (1991). We apply the method twice with testing level 0.05 and 0.01 , respectively, written as $\mathrm{Jo}(0.05)$ and $\mathrm{Jo}(0.01)$ in Table 1. The null-distribution of the trace test statistic is approximated by

$$
\left[\sum_{t=1}^{T} \boldsymbol{e}_{t}\left(\boldsymbol{X}_{t-1}-\overline{\boldsymbol{X}}\right)^{\prime}\right]\left[\sum_{t=1}^{T}\left(\boldsymbol{X}_{t-1}-\overline{\boldsymbol{X}}\right)\left(\boldsymbol{X}_{t-1}-\overline{\boldsymbol{X}}\right)^{\prime}\right]^{-1}\left[\sum_{t=1}^{T}\left(\boldsymbol{X}_{t-1}-\overline{\boldsymbol{X}}\right) \boldsymbol{e}_{t}^{\prime}\right],
$$

where $\boldsymbol{e}_{t}=\left(\varepsilon_{t, 1}, \ldots, \varepsilon_{t, p-r}\right)^{\prime}, \boldsymbol{X}_{0}=0$ and $\boldsymbol{X}_{t}=\sum_{j=1}^{t} \boldsymbol{e}_{t}$, and $\left\{\varepsilon_{t, i}\right\}$ are independent $N(0,1)$. See Johansen and Juselius (1990). This approximated distribution is calculated by simulation with $T=1000$ and 6000 replications. Due to the heavy computational burden, we have only considered the cases for $p \leq 30$.

Table 1 clearly indicates that the newly proposed method outperforms Johansen's method. The estimators $\widetilde{r}$ and $\widehat{r}^{*}$ defined in (2.7) and (4.4) achieve higher relative frequencies for attaining the true value $r$ than those 
achieved by the trace test with significance level at either 0.05 or 0.01 . For $n$ small, the situation becomes more challenging when $p$ and $r$ increase. For all $p \leq 30$, the new method works reasonably well even when $n=300$, and almost perfectly when $n \geq 500$. However, Johansen's method, which is not designed for large $p$, fails for $p \geq 20$ even when $n=2000$.

Example 2. Consider model (2.1) with a linear trend and one break point, i.e.,

$$
\begin{aligned}
& \mathbf{y}_{t}=\mathbf{a}_{1} I\left(0<t \leq t_{1}\right)+\mathbf{a}_{2} I\left(t_{1}<t \leq n\right)+\mathbf{b} t+\mathbf{x}_{t}, \\
& \mathbf{x}_{t}=\mathbf{A z}_{t}, t=1,2, \ldots, n,
\end{aligned}
$$

where the change point $t_{1}$ is taken as $[n / 2]$, the $i$-th component of $\mathbf{a}_{1}, \mathbf{a}_{2}$ and $\mathbf{b}$ are taken as $a_{1 i}=i / p, a_{2 i}=2+i / p$ and $b_{i}=0.5$ respectively, and $\mathbf{z}_{t 2}$ consists of $r$ stationary $\mathrm{AR}(1)$ processes with coefficients $-0.4+$ $i / r(i=1, \ldots, r)$, and let $p-r$ components of $\mathbf{z}_{t 1}$ be an $\operatorname{ARIMA}(1,1,1)$ with autoregressive coefficients generated independently from $U(0,0.6)$ and moving average coefficients $0.2+0.6 i /(p-r)(i=1, \ldots, p-r)$, and $\mathbf{A}$ is generated as in Example 1.

We estimate the cointegration rank $r$ by (2.7) with $\omega_{n}=\nu_{n}^{1 / 3}$ and (4.4) with $a_{n}=(n / q)^{1 / 2}, q=\left[n^{1 / 4}\right]$. We still take $M=50$ in $\widehat{\mathbf{W}}$ and replicate the exercise 500 times for each setting. The relative frequencies (RF) for 
Table 1: Relative frequencies (RF) of $\{\widehat{r}=r\}$ and average distance $D_{1}$ between the estimated and the true cointegration spaces (see (4.1)) in simulation with 500 replications for Example 1.

\begin{tabular}{|c|c|c|c|c|c|c|c|c|c|c|c|}
\hline & \multirow[b]{2}{*}{ Method } & \multicolumn{2}{|c|}{$n=300$} & \multicolumn{2}{|c|}{$n=500$} & \multicolumn{2}{|c|}{$n=1000$} & \multicolumn{2}{|c|}{$n=1500$} & \multicolumn{2}{|c|}{$n=2000$} \\
\hline & & $\overline{R F}$ & $D_{1}$ & $\mathrm{~F}$ & $D_{1}$ & $\mathrm{RF}$ & $D_{1}$ & $\overline{\mathrm{RF}}$ & $D_{1}$ & $\overline{R F}$ & $D_{1}$ \\
\hline \multirow{4}{*}{$\begin{array}{l}p=6 \\
r=1\end{array}$} & $0(0,05)$ & $\overline{170}$ & $\overline{1.656}$ & $\overline{28}$ & $\overline{c 606}$ & 0.424 & $\overline{0.456}$ & $\overline{4.408}$ & 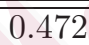 & .266 & $\overline{0.57}$ \\
\hline & & 406 & 0.467 & 460 & 0.420 & 0.692 & 0.258 & 666 & 0.285 & 0.536 & 0.362 \\
\hline & & 50 & 011 & $\overline{98}$ & $.0 t$ & 1.000 & 0.052 & ve & 0.004 & 1.000 & \\
\hline & & 888 & 0.1 & 2 & .07 & 0.990 & 0.058 & .990 & 0.005 & 1.000 & 0.045 \\
\hline \multirow{4}{*}{$\begin{array}{l}p=6 \\
r=3\end{array}$} & & $\overline{184}$ & 0.310 & 148 & .32 & 0.376 & 0.365 & .450 & $\overline{0.333}$ & 0.534 & $\overline{0.260}$ \\
\hline & & 730 & 0.185 & .706 & 0.191 & 0.646 & 0.222 & .706 & 0.200 & 0.796 & 0.126 \\
\hline & & & 0.06 & 00 & $\overline{0.0}$ & 00 & 0.06 & 2 & 0.000 & 1.000 & 0.029 \\
\hline & & 940 & 0.0 & 982 & 0.064 & 80 & 0.074 & 826 & 0.1 .18 & .000 & .029 \\
\hline \multirow{4}{*}{$\begin{array}{l}p=8 \\
r=3\end{array}$} & & 8 & 0.489 & $\overline{72}$ & 0.370 & $\overline{0.260}$ & 0.437 & 0.216 & $\overline{0.470}$ & $\overline{c 0.346}$ & $\overline{0.383}$ \\
\hline & & $\overline{404}$ & 0.352 & .694 & 0.207 & 0.536 & 0.283 & 0.484 & 0.310 & 0.610 & 0.233 \\
\hline & & 000 & $0.0 £$ & & 0.0 & 1.0 & 0.067 & 0 & 0.060 & 000 & 0.047 \\
\hline & & 188 & 0.1 & 922 & 0.11 & 0.912 & 0.112 & .924 & 0.100 & 0.994 & 0.050 \\
\hline \multirow{4}{*}{$\begin{array}{l}p=12 \\
r=6\end{array}$} & & 178 & 0.371 & 112 & 0.399 & $\overline{0.102}$ & $\overline{0.393}$ & $\overline{0.148}$ & $\overline{0.373}$ & $\overline{0.132}$ & 0.387 \\
\hline & & 584 & 0.224 & 466 & 0.253 & 0.452 & 0.247 & 0.530 & 0.211 & 0.448 & 0.242 \\
\hline & $I c$ & & 0.10 & & 00 & 1 & 0.058 & 0 & 040 & .000 & 0.041 \\
\hline & $\overline{\mathrm{AC}}$ & 622 & 0.210 & .906 & 0.105 & 0.942 & 0.078 & 0.982 & 0.046 & 0.988 & 0.04 \\
\hline \multirow{4}{*}{$\begin{array}{l}p=20 \\
r=14\end{array}$} & & 32 & 0.289 & .102 & 0.286 & 0.148 & $\overline{0.263}$ & $\overline{0.294}$ & $\overline{0.210}$ & 0.232 & 0.237 \\
\hline & & 74 & 0.202 & 456 & 0.187 & 0.554 & 0.155 & 0.748 & 0.097 & 0.596 & 0.134 \\
\hline & $I C$ & & & & 0. & 00 & 54 & 00 & 039 & 00 & 0.039 \\
\hline & $\mathrm{ACF}$ & 0.652 & 0.169 & 0.928 & 0.084 & 0.964 & 0.061 & 0.990 & 0.041 & 0.992 & 0.041 \\
\hline \multirow{4}{*}{$\begin{array}{l}p=30 \\
r=20\end{array}$} & & 34 & 0.314 & 0.074 & 0.297 & 0.170 & $\overline{0.249}$ & 0.066 & $\overline{0.283}$ & 0.106 & 0.265 \\
\hline & & 30 & 0.272 & 0.254 & 0.239 & 0.436 & 0.186 & 0.234 & 0.221 & 0.340 & 0.193 \\
\hline & $I C$ & 94 & 0.240 & 0.944 & 0.1 & 1.000 & 0.096 & 1.000 & 0.079 & 1.000 & 0.067 \\
\hline & . & 0.072 & 0.320 & 0.498 & 0.203 & 0.832 & 0.122 & 0.854 & 0.106 & 0.896 & 0.087 \\
\hline
\end{tabular}

Herein, Jo(0.05) and Jo(0.01) are based on Johansen's method with levels 0.05 and 0.01, respectively, IC is based on the proposed method and ACF is based on (4.4) by ZRY, $r$ is the cointegration rank.

the occurrence of the event $\{\widetilde{r}=r\}$ or $\{\widehat{r}=r\}$ and the average value of $D_{1}=D_{1}^{*}\left(\mathcal{M}\left(\widehat{\mathbf{A}}_{2}\right), \mathcal{M}\left(\mathbf{A}_{2}\right)\right)$ over 500 replications for $t_{1}=[n / 2]$ are listed in Table 2 under the labels IC and ACF. It is shown from Table 2 that both proposed procedures work reasonably well when $p$ is small and the 
sample size $n$ is relatively large. When $p$ is large, for example $p \geq 50$, the ACF method works poorly, but the proposed method can still estimate the cointegration rank and the cointegration space very well. This also confirms that ZRY's method works for smaller $p$ than the proposed method.

Also included in Table 2 are the results of applying the Phillips-Perron unit-root test (PP.test) to the process $\left\{\widehat{\mathbf{z}}_{t}\right\}$ defined in (4.2), with significance level set at 0.01 , for estimating $r$. When $p$ is large and $n$ is small, the PP.test estimates $r$ slightly better than the procedures (4.4) but worse than (2.7). In all cases, (2.7) always performs better than the other methods. Also noticeable in Table 2 is the fact that for a given $p$, the larger $r / p$ is, the more accurate are the estimates for $r$. In general, (2.7) performs more stably than unit-root test procedures based on PP.test and (4.4).

To give some insight of the underpinning logic, we present the box plots of $D_{1}^{*}\left(\mathcal{M}\left(\widehat{\mathbf{A}}_{2}\right), \mathcal{M}\left(\mathbf{A}_{2}\right)\right)$ based on (2.7) and PP.test for $(p, r)=(6,2)$ and $(8,4)$ in Figure 1 and Figure 2 respectively. From Figures 1 and 2, it is shown that both the information criterion and PP.test work well and the estimation errors decrease as sample size $n$ increases.

Example 3. Consider model (2.1) with one change point, i.e.,

$$
\mathbf{y}_{t}=\mathbf{a}_{1} I\left(0<t \leq t_{1}\right)+\mathbf{a}_{2} I\left(t_{1}<t \leq n\right)+\mathbf{b} t+\mathbf{A} \mathbf{z}_{t}
$$

where the change point $t_{1}$ is taken as $[n / 2]$, the coefficients $\mathbf{a}_{1}, \mathbf{a}_{2}, \mathbf{b}$ and 
Table 2: relative frequencies (RF) of $\{\widehat{r}=r\}$ and average distance $D_{1}$ between the estimated and the true cointegration spaces in simulation with 500 replications for Example 2.

\begin{tabular}{|c|c|c|c|c|c|c|c|c|c|c|c|c|c|}
\hline \multirow[b]{2}{*}{$(p, r)$} & \multirow[b]{2}{*}{ Method } & \multicolumn{2}{|c|}{$n=300$} & \multicolumn{2}{|c|}{$n=500$} & \multicolumn{2}{|c|}{$n=1000$} & \multicolumn{2}{|c|}{$n=1500$} & \multicolumn{2}{|c|}{$n=2000$} & \multicolumn{2}{|c|}{$n=2500$} \\
\hline & & $\mathrm{RF}$ & $D_{1}$ & RF & $D_{1}$ & RF & $D_{1}$ & $\overline{R F}$ & $D_{1}$ & RF & $D_{1}$ & $\mathrm{RF}$ & $D_{1}$ \\
\hline$(6,2)$ & $\mathrm{ACF}$ & 0.880 & 0.151 & 0.936 & 0.119 & 0.950 & 0.098 & 0.988 & 0.061 & 0.968 & 0.071 & 0.952 & 0.085 \\
\hline \multirow{3}{*}{$(6,3)$} & $I C$ & 0.980 & 0.088 & 0.998 & 0.066 & 1.000 & 0.063 & 1.000 & 0.038 & 1.000 & 0.060 & 1.000 & 0.070 \\
\hline & $\mathrm{ACF}$ & 0.892 & 0.129 & 0.964 & 0.083 & 0.908 & 0.110 & 0.996 & 0.040 & 0.814 & 0.161 & 0.754 & 0.202 \\
\hline & PP.test & 0.916 & 0.131 & 0.972 & 0.084 & 0.954 & 0.092 & 0.992 & 0.042 & 0.914 & 0.117 & 0.928 & 0.118 \\
\hline$(8,3)$ & PP.test & 0.918 & 0.138 & 0.976 & 0.091 & 0.958 & 0.089 & 0.994 & 0.053 & 0.994 & 0.057 & 0.944 & 0.088 \\
\hline \multirow{3}{*}{$(8,4)$} & $I C$ & 0.996 & 0.075 & 1.000 & 0.060 & 1.000 & 0.049 & 1.000 & 0.055 & 1.000 & 0.041 & 1.000 & 0.029 \\
\hline & $\mathrm{ACF}$ & 0.896 & 0.113 & 0.970 & 0.072 & 0.980 & 0.057 & 0.956 & 0.075 & 0.996 & 0.042 & 1.000 & 0.029 \\
\hline & PP.test & 0.986 & 0.081 & 0.998 & 0.061 & 0.990 & 0.053 & 0.982 & 0.065 & 0.992 & 0.044 & 0.996 & 0.031 \\
\hline \multirow{2}{*}{$(12,6)$} & $\overline{I C}$ & 1.000 & 0.101 & 1.000 & 0.074 & 1.000 & 0.050 & 1.000 & 0.051 & 1.000 & 0.043 & 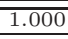 & 0.033 \\
\hline & $\mathrm{ACF}$ & 0.762 & 0.170 & 0.918 & 0.100 & 0.990 & 0.053 & 0.960 & 0.065 & 0.980 & 0.050 & 0.998 & 0.034 \\
\hline \multirow{3}{*}{$(20,14)$} & $I C$ & 0.942 & 0.125 & 0.996 & 0.080 & 1.000 & 0.055 & 1.000 & 0.045 & 1.000 & 0.043 & 1.000 & 0.037 \\
\hline & $\mathrm{ACF}$ & 0.604 & 0.180 & 0.894 & 0.099 & 0.964 & 0.064 & 0.960 & 0.054 & 0.940 & 0.057 & 0.942 & 0.052 \\
\hline & PP.test & 0.908 & 0.152 & 0.990 & 0.083 & 0.990 & 0.059 & 0.986 & 0.054 & 0.978 & 0.054 & 0.990 & 0.045 \\
\hline \multirow{3}{*}{$(30,20)$} & $I C$ & 0.826 & 0.226 & 0.988 & 0.151 & 1.000 & 0.102 & 1.000 & 0.082 & 1.000 & 0.071 & 1.000 & 0.059 \\
\hline & $\mathrm{ACF}$ & 0.114 & 0.315 & 0.386 & 0.237 & 0.696 & 0.155 & 0.796 & 0.121 & 0.772 & 0.121 & 0.894 & 0.081 \\
\hline & PP.test & 0.532 & 0.330 & 0.642 & 0.300 & 0.878 & 0.158 & 0.934 & 0.112 & 0.928 & 0.106 & 0.984 & 0.065 \\
\hline \multirow{3}{*}{$(50,30)$} & $\overline{I C}$ & 0.128 & 0.448 & 0.520 & 0.355 & 0.996 & 0.233 & 1.000 & 0.189 & 1.000 & 0.143 & 1.000 & 0.134 \\
\hline & $\mathrm{ACF}$ & 0.018 & 0.481 & 0.084 & 0.419 & 0.048 & 0.441 & 0.046 & 0.435 & 0.118 & 0.326 & 0.030 & 0.427 \\
\hline & PP.test & 0.014 & 0.766 & 0.014 & 0.775 & 0.048 & 0.690 & 0.140 & 0.587 & 0.490 & 0.361 & 0.310 & 0.478 \\
\hline \multirow{3}{*}{$(80,50)$} & $I C$ & 0.160 & 0.505 & 0.064 & 0.459 & 0 & 0.371 & 0.392 & 0.295 & 0.930 & 0.245 & 0.998 & 0.223 \\
\hline & $\mathrm{ACF}$ & 0 & 0.539 & 0.052 & 0.474 & 0.044 & 0.461 & 0.002 & 0.544 & 0 & 0.589 & 0 & 0.660 \\
\hline & PP.test & 0.016 & 0.675 & 0 & 0.799 & 0.004 & 0.796 & 0.002 & 0.812 & 0 & 0.800 & 0.002 & 0.823 \\
\hline
\end{tabular}

IC is based on the proposed method, ACF is based on (4.4) by ZRY, PP.test is based on Phillips-Perron unit-root test and $r$ is the cointegration rank.

$\mathbf{z}_{t 2}$ are taken as Example 2. We only change the autoregressive coefficients in the components of $\mathbf{z}_{t 1}$, which are taken as $0.3+(-1)^{i} 0.5 i /(p-r), i=$ $1, \ldots, p-r$. The cointegration rank $r$ is estimated as Example 2. Based on 500 replications, we report the relative frequencies (RF) for the occurrence of the event $\{\widetilde{r}=r\}$ or $\{\widehat{r}=r\}$ and the average value of $D_{1}=$ $D_{1}^{*}\left(\mathcal{M}\left(\widehat{\mathbf{A}}_{2}\right), \mathcal{M}\left(\mathbf{A}_{2}\right)\right)$ in Table 3 , It can be seen in Table 3 that the pro- 

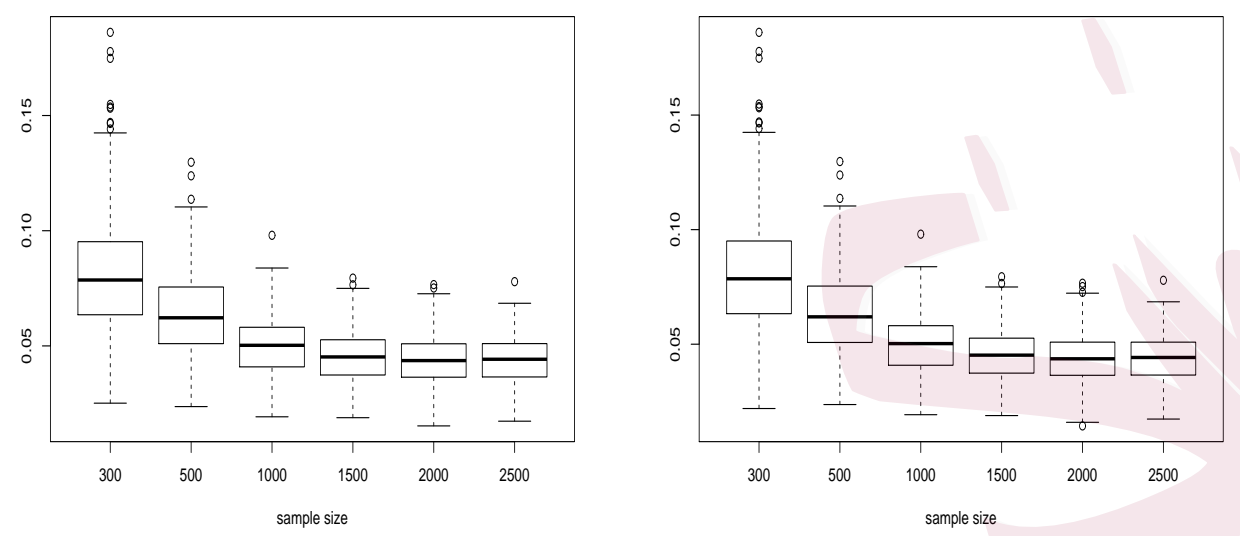

Figure 1: The left panel is the box plot of the distance $D_{1}^{*}\left(\mathcal{M}\left(\widehat{\mathbf{A}}_{2}\right), \mathcal{M}\left(\mathbf{A}_{2}\right)\right)$ based on the proposed method and the right panel is based on PP.test when $p=6, r=2$ in Example 2.
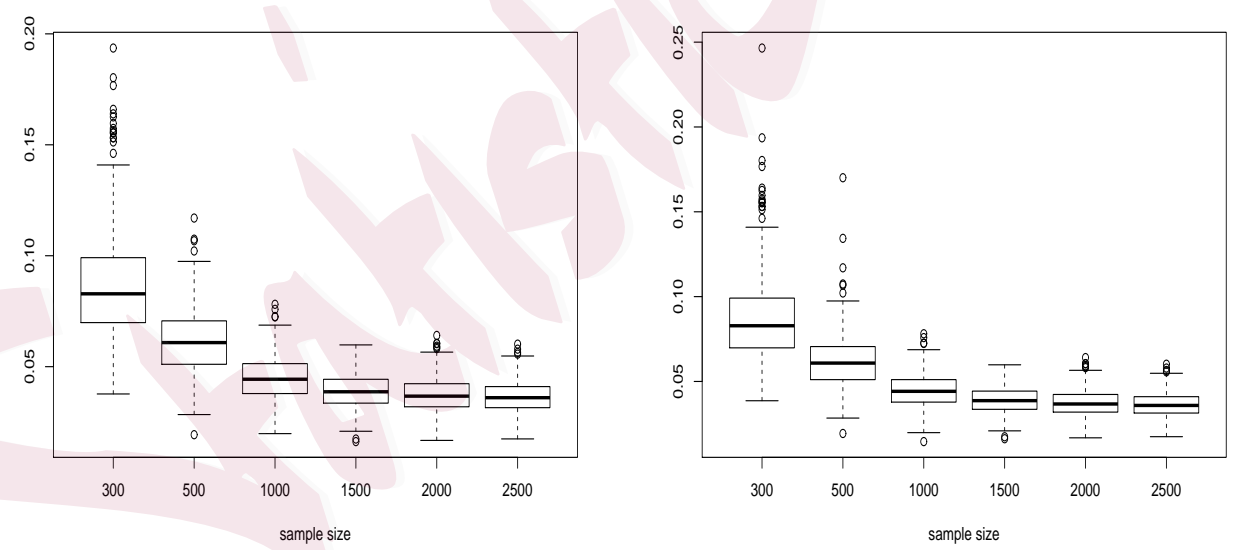

Figure 2: The left panel is the box plot of the distance $D_{1}^{*}\left(\mathcal{M}\left(\widehat{\mathbf{A}}_{2}\right), \mathcal{M}\left(\mathbf{A}_{2}\right)\right)$ based on the proposed method and the right panel is based on PP.test when $p=8, r=4$ in Example 2.

posed procedure based on (2.7) works reasonably well, especially when $p$ is small or when the sample size $n$ is large. Further, combining with Example 
2, it can be seen that the unit-root procedures based on PP.test or (4.4) are not stable and are affected by the coefficients of the nonstationary process, but that (2.7) performs reasonably well in general.

Table 3: Relative frequencies (RF) of $\{\widehat{r}=r\}$ and average distance $D_{1}$ between the estimated and the true cointegration spaces in simulation with 500 replications for Example 3.

\begin{tabular}{|c|c|c|c|c|c|c|c|c|c|c|c|c|c|}
\hline \multirow[b]{2}{*}{$(p, r)$} & \multirow[b]{2}{*}{ Method } & \multicolumn{2}{|c|}{$n=300$} & \multicolumn{2}{|c|}{$n=500$} & \multicolumn{2}{|c|}{$n=1000$} & \multicolumn{2}{|c|}{$n=1500$} & \multicolumn{2}{|c|}{$n=2000$} & \multicolumn{2}{|c|}{$n=2500$} \\
\hline & & $\mathrm{RF}$ & $D_{1}$ & $\mathrm{RF}$ & $D_{1}$ & $\mathrm{RF}$ & $D_{1}$ & $\mathrm{RF}$ & $D_{1}$ & $\mathrm{RF}$ & $D_{1}$ & $\mathrm{RF}$ & $D_{1}$ \\
\hline \multirow{3}{*}{$(6,2)$} & $\overline{I C}$ & 0.994 & 0.120 & 0.998 & $\overline{0.099}$ & 1.000 & 0.088 & 1.000 & 0.085 & 1.000 & 0.082 & 1.000 & 0.083 \\
\hline & $\mathrm{ACF}$ & 0.454 & 0.430 & 0.398 & 0.461 & 0.282 & 0.529 & 0.266 & 0.539 & 0.208 & 0.575 & 0.158 & 0.607 \\
\hline & PP.test & 0.412 & 0.461 & 0.428 & 0.443 & 0.370 & 0.474 & 0.358 & 0.480 & 0.314 & 0.507 & 0.264 & 0.539 \\
\hline \multirow{3}{*}{$(6,3)$} & $\overline{I C}$ & $\overline{0.990}$ & $\overline{0.092}$ & 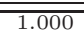 & $\overline{0.071}$ & 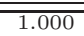 & $\overline{0.059}$ & 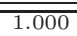 & $\overline{0.054}$ & $\bar{~} 1.000$ & $\overline{0.052}$ & 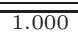 & $\overline{0.051}$ \\
\hline & $\mathrm{ACF}$ & 0.840 & 0.157 & 0.896 & 0.123 & 0.850 & 0.137 & 0.820 & 0.147 & 0.786 & 0.169 & 0.718 & 0.207 \\
\hline & PP.test & 0.936 & 0.127 & 0.946 & 0.106 & 0.906 & 0.123 & 0.912 & 0.116 & 0.898 & 0.122 & 0.872 & 0.139 \\
\hline \multirow{3}{*}{$(8,3)$} & $\overline{I I C}$ & $\overline{\overline{0.996}}$ & $\overline{0.116}$ & 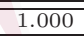 & $\overline{0.087}$ & 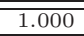 & $\overline{0.071}$ & 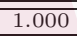 & 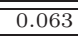 & "1.000 & $\overline{c 0.060}$ & 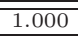 & 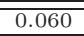 \\
\hline & $\mathrm{ACF}$ & 0.692 & 0.244 & 0.780 & 0.197 & 0.720 & 0.221 & 0.668 & 0.244 & 0.600 & 0.282 & 0.562 & 0.308 \\
\hline & PP.test & 0.848 & 0.206 & 0.836 & 0.188 & 0.830 & 0.185 & 0.828 & 0.179 & 0.804 & 0.192 & 0.770 & 0.216 \\
\hline \multirow{3}{*}{$(8,4)$} & $I C$ & 0.998 & 0.105 & 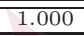 & 0.087 & 1.000 & $\overline{0.079}$ & 1.000 & 0.075 & 21.000 & 0.074 & 1.000 & 0.072 \\
\hline & $\mathrm{ACF}$ & 0.368 & 0.415 & 0.258 & 0.489 & 0.148 & 0.579 & 0.116 & 0.602 & 0.076 & 0.657 & 0.080 & 0.658 \\
\hline & PP.test & 0.396 & 0.537 & 0.336 & 0.571 & 0.264 & 0.636 & 0.236 & 0.652 & 0.192 & 0.690 & 0.214 & 0.673 \\
\hline \multirow{3}{*}{$(12,6)$} & $\overline{I I C}$ & $\overline{0.998}$ & 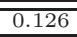 & 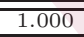 & $\overline{0.097}$ & 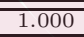 & $\overline{0.076}$ & 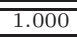 & $\overline{0.069}$ & 1.000 & $\overline{c 0.066}$ & 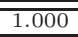 & 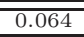 \\
\hline & $\mathrm{ACF}$ & 0.384 & 0.358 & 0.330 & 0.395 & 0.182 & 0.485 & 0.130 & 0.534 & 0.080 & 0.581 & 0.052 & 0.622 \\
\hline & PP.test & 0.418 & 0.509 & 0.418 & 0.521 & 0.324 & 0.588 & 0.304 & 0.615 & 0.250 & 0.667 & 0.248 & 0.665 \\
\hline \multirow{3}{*}{$(12,8)$} & $I C$ & $\overline{0.992}$ & 0.095 & 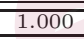 & $\overline{0.075}$ & $\overline{1.000}$ & $\overline{0.063}$ & 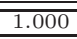 & 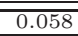 & 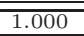 & $\overline{c 0.056}$ & 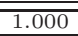 & $\overline{c 0.056}$ \\
\hline & $\mathrm{ACF}$ & 0.398 & 0.322 & 0.292 & 0.381 & 0.168 & 0.456 & 0.120 & 0.477 & 0.084 & 0.513 & 0.060 & 0.561 \\
\hline & PP.test & 0.468 & 0.485 & 0.378 & 0.570 & 0.312 & 0.635 & 0.254 & 0.690 & 0.242 & 0.700 & 0.206 & 0.740 \\
\hline \multirow{3}{*}{$(20,14)$} & 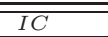 & $\overline{\overline{0.894}}$ & $\overline{0.161}$ & 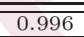 & $\overline{\overline{0.112}}$ & $\overline{\overline{1.000}}$ & $\overline{\overline{0.079}}$ & 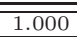 & 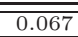 & $\bar{~} 1.000$ & $\overline{c 0.061}$ & 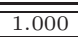 & $\overline{0.057}$ \\
\hline & $\mathrm{ACF}$ & 0.284 & 0.331 & 0.306 & 0.330 & 0.212 & 0.366 & 0.182 & 0.396 & 0.128 & 0.437 & 0.088 & 0.471 \\
\hline & PP.test & 0.340 & 0.490 & 0.380 & 0.489 & 0.382 & 0.530 & 0.360 & 0.572 & 0.290 & 0.641 & 0.270 & 0.647 \\
\hline \multirow{3}{*}{$(30,20)$} & $\overline{I I C}$ & $\overline{\overline{0.706}}$ & $\overline{0.274}$ & 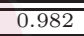 & $\overline{\overline{0.196}}$ & $\overline{1.000}$ & $\overline{0.135}$ & 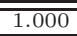 & $\overline{\overline{0.111}}$ & 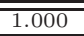 & $\overline{c 0.096}$ & 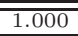 & $\overline{c 0.087}$ \\
\hline & $\mathrm{ACF}$ & 0.178 & 0.380 & 0.132 & 0.402 & 0.120 & 0.405 & 0.074 & 0.423 & 0.068 & 0.431 & 0.082 & 0.438 \\
\hline & PP.test & 0.112 & 0.624 & 0.160 & 0.582 & 0.258 & 0.513 & 0.250 & 0.546 & 0.250 & 0.559 & 0.290 & 0.539 \\
\hline \multirow{3}{*}{$(50,30)$} & 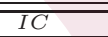 & 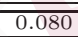 & 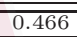 & 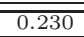 & $\overline{\overline{0.392}}$ & $\overline{0.988}$ & $\overline{\overline{0.281}}$ & 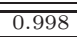 & 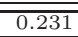 & $\bar{~} 1.000$ & 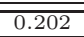 & 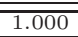 & 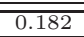 \\
\hline & $\mathrm{ACF}$ & 0.104 & 0.492 & 0.068 & 0.520 & 0.002 & 0.603 & 0.002 & 0.613 & 0 & 0.618 & 0.002 & 0.620 \\
\hline & PP.test & 0.006 & 0.812 & 0 & 0.835 & 0.002 & 0.835 & 0.010 & 0.787 & 0.010 & 0.765 & 0.014 & 0.750 \\
\hline \multirow{3}{*}{$(80,50)$} & $\overline{I I C}$ & $\overline{0.312}$ & $\overline{0.510}$ & $\overline{0.024}$ & $\overline{0.470}$ & $\overline{0.036}$ & $\overline{\overline{0.390}}$ & 0.662 & $\overline{0.327}$ & 0.978 & 0.288 & 0.998 & $\begin{array}{c}0.263 \\
\end{array}$ \\
\hline & $\mathrm{ACF}$ & 0.002 & 0.521 & 0.092 & 0.510 & 0 & 0.633 & 0 & 0.691 & 0 & 0.724 & 0 & 0.740 \\
\hline & PP.test & 0 & 0.833 & 0.002 & 0.828 & 0 & 0.890 & 0 & 0.892 & 0 & 0.881 & 0 & 0.876 \\
\hline
\end{tabular}

IC is based on the proposed method, ACF is based on (4.4) by ZRY, PP.test is based on Phillips-Perron unit-root test and $r$ is the cointegration rank.

To demonstrate that the proposed method is more robust than that based on the unit-root test, we also present the box plots of $D_{1}^{*}\left(\mathcal{M}\left(\widehat{\mathbf{A}}_{2}\right), \mathcal{M}\left(\mathbf{A}_{2}\right)\right)$ based on (2.7) and PP.test for $(p, r)=(8,4)$ and $(12,6)$ in Figure 3 and 
Figure 4 respectively. From these figures, it can be seen that the estimation errors decrease as sample size $n$ increases for both procedures. However, the information criterion always performs better than PP.test.
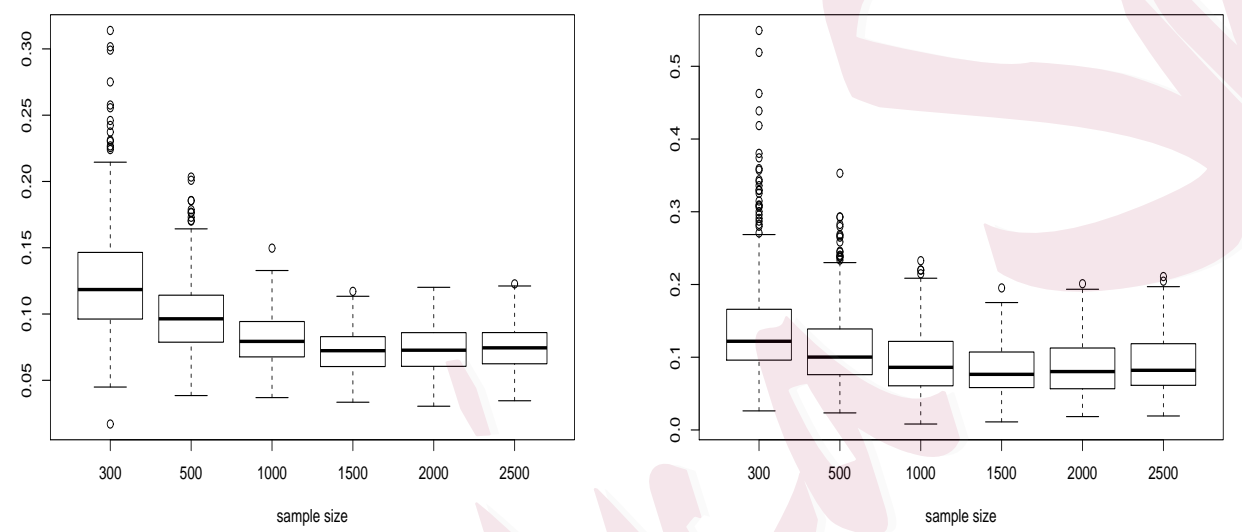

Figure 3: The left panel is the box plot of the distance $D_{1}^{*}\left(\mathcal{M}\left(\widehat{\mathbf{A}}_{2}\right), \mathcal{M}\left(\mathbf{A}_{2}\right)\right)$ based on the proposed method and the right panel is based on PP.test when $p=8, r=4$ in Example 3 .

Example 4. Now we consider an example in which the components of $\mathbf{y}_{t}$ are $I(1)$ with a constant trend and one break point, i.e.,

$$
\begin{aligned}
\mathbf{y}_{t} & =\boldsymbol{a}_{1} I\left(0<t \leq t_{1}\right)+\boldsymbol{a}_{2} I\left(t_{1}<t \leq n\right)+\boldsymbol{X}_{t} \\
& =\boldsymbol{a}_{1} I\left(0<t \leq t_{1}\right)+\boldsymbol{a}_{2} I\left(t_{1}<t \leq n\right)+\mathbf{A z}_{t}
\end{aligned}
$$

where $\mathbf{a}_{1}, \mathbf{a}_{2}$ and $\mathbf{z}_{t}$ are given as in Example 3. Table 4 reports the relative frequencies of the occurrence of the event $\{\widehat{r}=r\}$ and the average distance (4.1) for the case with a change point $t_{1}=[n / 2]$ in a simulation with 500 

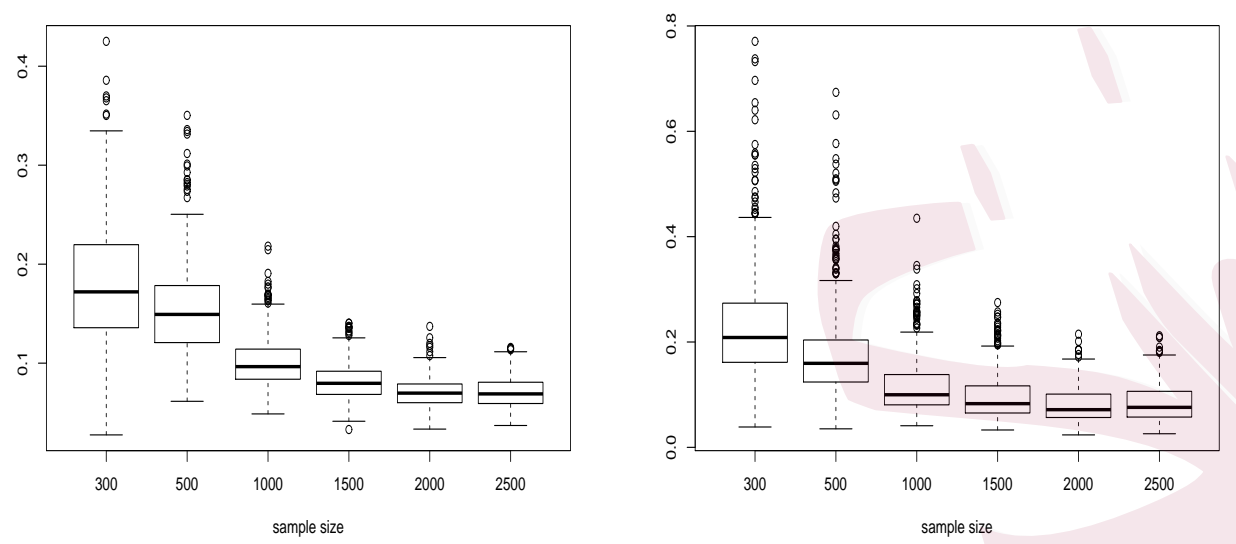

Figure 4: The left panel is the box plot of the distance $D_{1}^{*}\left(\mathcal{M}\left(\widehat{\mathbf{A}}_{2}\right), \mathcal{M}\left(\mathbf{A}_{2}\right)\right)$ based on the proposed method and the right panel is based on PP.test when $p=12, r=6$ in Example 3.

replications, where the cointegration rank is estimated as Example 2. Also included in Table 4 are the results obtained from applying the PhillipsPerron unit-root test for $\widehat{\mathbf{z}}_{t}$ defined in (4.2) to estimate $r$. Table 4 indicates that (2.7) works well even in the presence of breaks in a constant trend. However, the unit-root procedure performs very poorly if one overlooks the breaks in the data.

Example 5. Next, we consider an example with breaks in level and slope.

Let

$$
\begin{aligned}
\mathbf{y}_{t} & =\left(\boldsymbol{a}_{1}+\mathbf{b}_{1} t\right) I\left(0<t \leq t_{1}\right)+\left(\boldsymbol{a}_{2}+\mathbf{b}_{2} t\right) I\left(t_{1}<t \leq n\right)+\boldsymbol{X}_{t} \\
& =\left(\boldsymbol{a}_{1}+\mathbf{b}_{1} t\right) I\left(0<t \leq t_{1}\right)+\left(\boldsymbol{a}_{2}+\mathbf{b}_{2} t\right) I\left(t_{1}<t \leq n\right)+\mathbf{A} \mathbf{z}_{t},
\end{aligned}
$$


Table 4: Relative frequencies (RF) of $\{\hat{r}=r\}$ and average distance $D_{1}$ between the estimated and the true cointegration spaces in simulation with 500 replications for Example 4.

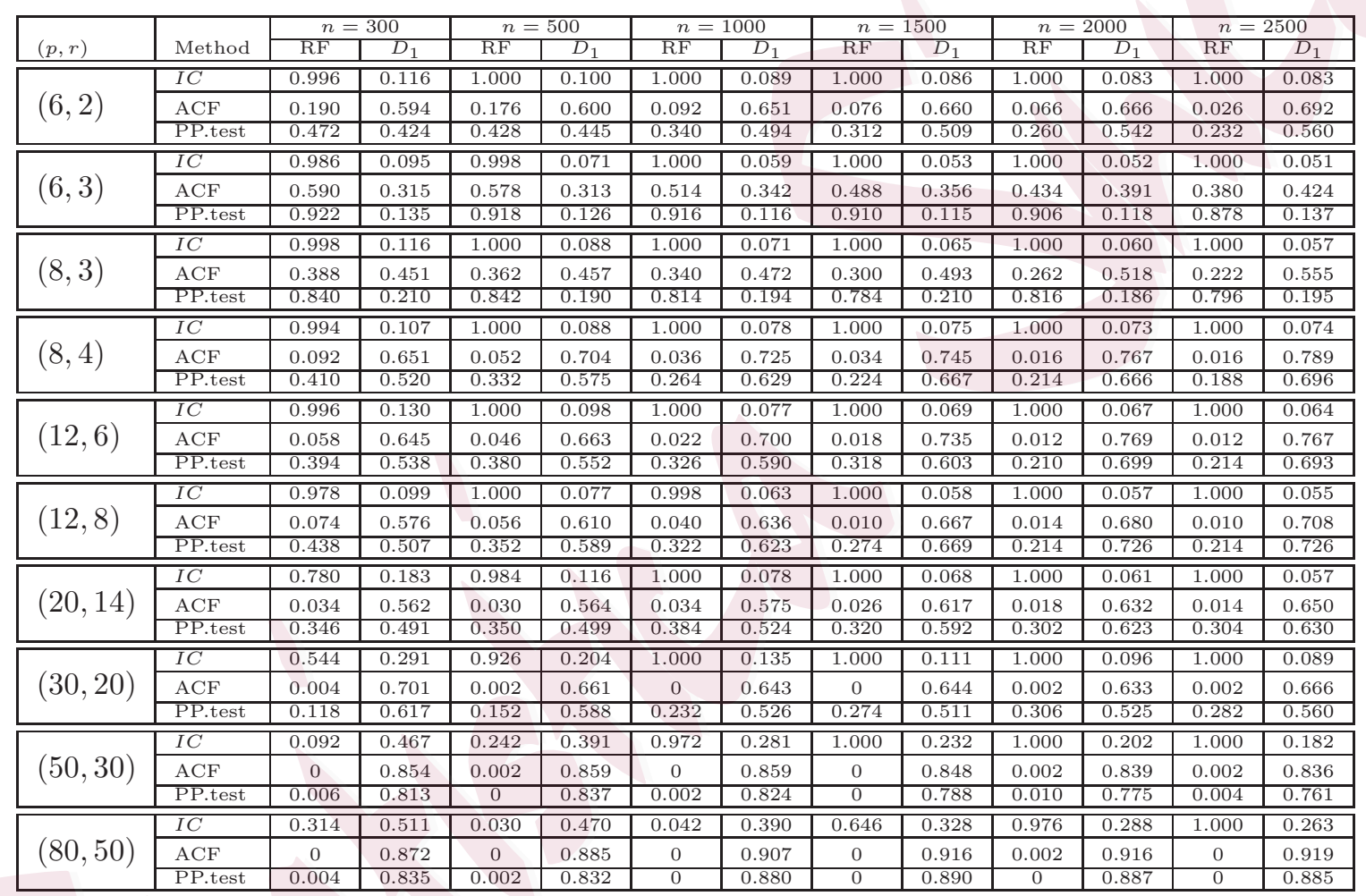

IC is based on the proposed method, ACF is based on (4.4) by ZRY, PP.test is based on Phillips-Perron unit-root test and $r$ is the cointegration rank.

where $\mathbf{a}_{1}, \mathbf{a}_{2}, \mathbf{z}_{t}$ are given as in Example $3, t_{1}=[n / 2]$ and all the compo-

nents of $\mathbf{b}_{1}$ and $\mathbf{b}_{2}$ are taken as 0.3 and 0.8 respectively. It follows from

(4.5) that

$$
\begin{aligned}
\nabla \mathbf{y}_{t}= & \mathbf{b}_{1} I\left(1<t \leq t_{1}\right)+\left\{\mathbf{b}_{2}+\left(\mathbf{b}_{2}-\mathbf{b}_{1}\right) t_{1}\right\} I\left(t=t_{1}+1\right) \\
& +\mathbf{b}_{2} I\left(t>t_{1}+1\right)+\nabla \mathbf{z}_{t} .
\end{aligned}
$$


We estimate the change point $t_{1}$ by

$$
\widehat{t_{1}}=\operatorname{argmax}_{1 \leq t \leq n}\left|\nabla y_{t, 2}\right|
$$

and then apply our procedure to estimate $r$ and the cointegration space by replacing $\nabla \mathbf{y}_{\widehat{t}_{1}+1}$ with zero. The corresponding relative frequencies of the occurrence of the event $\{\widehat{r}=r\}$ and the average distance (4.1) in a simulation with 500 replications are reported in Table 5. Table 5 indicates that the proposed (2.7) procedure is simple to carry out and works well even in the presence of breaks in a linear trend.

Table 5: Relative frequencies (RF) of $\{\widehat{r}=r\}$ and average distance $D_{1}$ between the estimated and the true cointegration spaces by the proposed method in simulation with 500 replications for Example 5.

\begin{tabular}{|c|c|c|c|c|c|c|c|c|c|c|c|c|}
\hline & $n=$ & 300 & $n=$ & 500 & $n=$ & 000 & $n=$ & 1500 & & 000 & $n=$ & 2500 \\
\hline$(p, r)$ & $\mathrm{RF}$ & $D_{1}$ & $\mathrm{RF}$ & $D_{1}$ & $\mathrm{RF}$ & $D_{1}$ & $\mathrm{RF}$ & $D_{1}$ & $\mathrm{RF}$ & $D_{1}$ & $\mathrm{RF}$ & $D_{1}$ \\
\hline$(6,3)$ & 0.992 & 0.090 & 1.000 & 0.070 & 1.000 & 0.058 & 1.000 & 0.054 & 1.000 & 0.051 & 1.000 & 0.051 \\
\hline$(8,4)$ & 0.996 & 0.105 & 1.000 & 0.088 & 1.000 & 0.078 & 1.000 & 0.076 & 1.000 & 0.073 & 1.000 & 0.073 \\
\hline$(12,8)$ & 0.996 & 0.091 & 1.000 & 0.074 & 1.000 & 0.062 & 1.000 & 0.058 & 1.000 & 0.057 & 1.000 & 0.055 \\
\hline$(20,14)$ & 0.936 & 0.158 & 0.996 & 0.111 & 1.000 & 0.079 & 1.000 & 0.067 & 1.000 & 0.060 & 1.000 & 0.057 \\
\hline$(30,20)$ & 0.820 & 0.272 & 0.980 & 0.198 & 1.000 & 0.135 & 1.000 & 0.112 & 1.000 & 0.096 & 1.000 & 0.087 \\
\hline$(50,30)$ & 0.024 & 0.473 & 0.088 & 0.397 & 0.966 & 0.283 & 1.000 & 0.233 & 1.000 & 0.202 & 1.000 & 0.183 \\
\hline$(80,50)$ & 0.246 & 0.512 & 0.004 & 0.474 & 0.010 & 0.394 & 0.580 & 0.330 & 0.972 & 0.290 & 1.000 & 0.264 \\
\hline
\end{tabular}

In summary, it is seen from Examples 1-5 that the proposed (2.7) procedure works reasonably well when $p$ is small and $n$ is large. When $p$ is large, for example when $p \geq 50$, methods based on the ACF of ZRY, the likelihood ratio of Johansen and the PP.test all fail to work adequately. The proposed method, however, can still estimate the cointegration rank 
and the cointegration space very well for large $p$. That is, (2.7) always performs better than the other methods. It is shown that for a given $p$, the larger $r / p$ is, the more accurate are the proposed estimates for $r$. In general, (2.7) is more robust than other existing procedures. Both the figures and tables show that the estimation errors of the proposed method decreases as the sample size $n$ increases. There are a couple of possible explanations for the superiority of the proposed method. First, the breaks have little effect on estimating the cointegration rank when $\nabla \mathbf{y}_{t}-\nabla \overline{\mathbf{y}}$ is used, while other procedures using the original series are sensitive to the existence of breaks. The advantage of our method is that it avoids estimating and testing for the trends. Second, the proposed method allows a larger $p$ since the matrix for recovering cointegration space is based on the stationary process $\nabla \mathbf{y}_{t}$, while other methods can only deal with smaller values of $p$.

Example 6. We consider the monthly exchange rates of the U.S. dollar versus the currencies of 19 OECD countries (Austria, Belgium, Canada, Denmark, Finland, France, Germany, Ireland, Italy, Japan, Korea, Netherlands, Norway, Portugal, South Africa, Spain, Sweden, Switzerland, and the United Kingdom) from January 1973 to December 2000 published by the Federal Reserve Economic Data (FRED), which were analyzed in Engle, Mark and West (2015) and Trapani (2021). All the exchange rates are 
transformed by taking logarithm, each of the transformed series are shown to follow a unit-root model based on PP.test at significance levels 0.05 and 0.1. Applying the proposed method of this paper to the transformed data, both the (4.4) and the PP.test at significance level 0.1 for the series $\widehat{\mathbf{z}}_{t}=\widehat{\mathbf{A}}^{\prime} \mathbf{x}_{t}$ lead to $\widehat{r}=2$ with $M=n^{1 / 4} ; \widehat{r}=4$ with $M=4(n / 100)^{1 / 4}$ (given as in KPSS test); and $\widehat{r}=1$ for both $M=n^{1 / 4}$ and $M=4(n / 100)^{1 / 4}$ by the PP.test at significance level 0.05. To shed further light on the estimated rank, we plot the four possible cointegrated series and their ACF corresponding to the first four smallest eigenvalues based on (4.4) with $M=4(n / 100)^{1 / 4}$ and $M=n^{1 / 4}$ in Figure 5 and Figure 6 respectively. Both figures show that two estimated series are stationary, while the other two series tend to be nonstationary. It would therefore be more reasonable to estimate the cointegration rank as 2 . On the other hand, all procedures show that cointegration exists in the exchange rates, which is consistent with the findings of Engle, Mark and West (2015), who showed that these exchange rates can be modeled by a nonstationary factor model. 

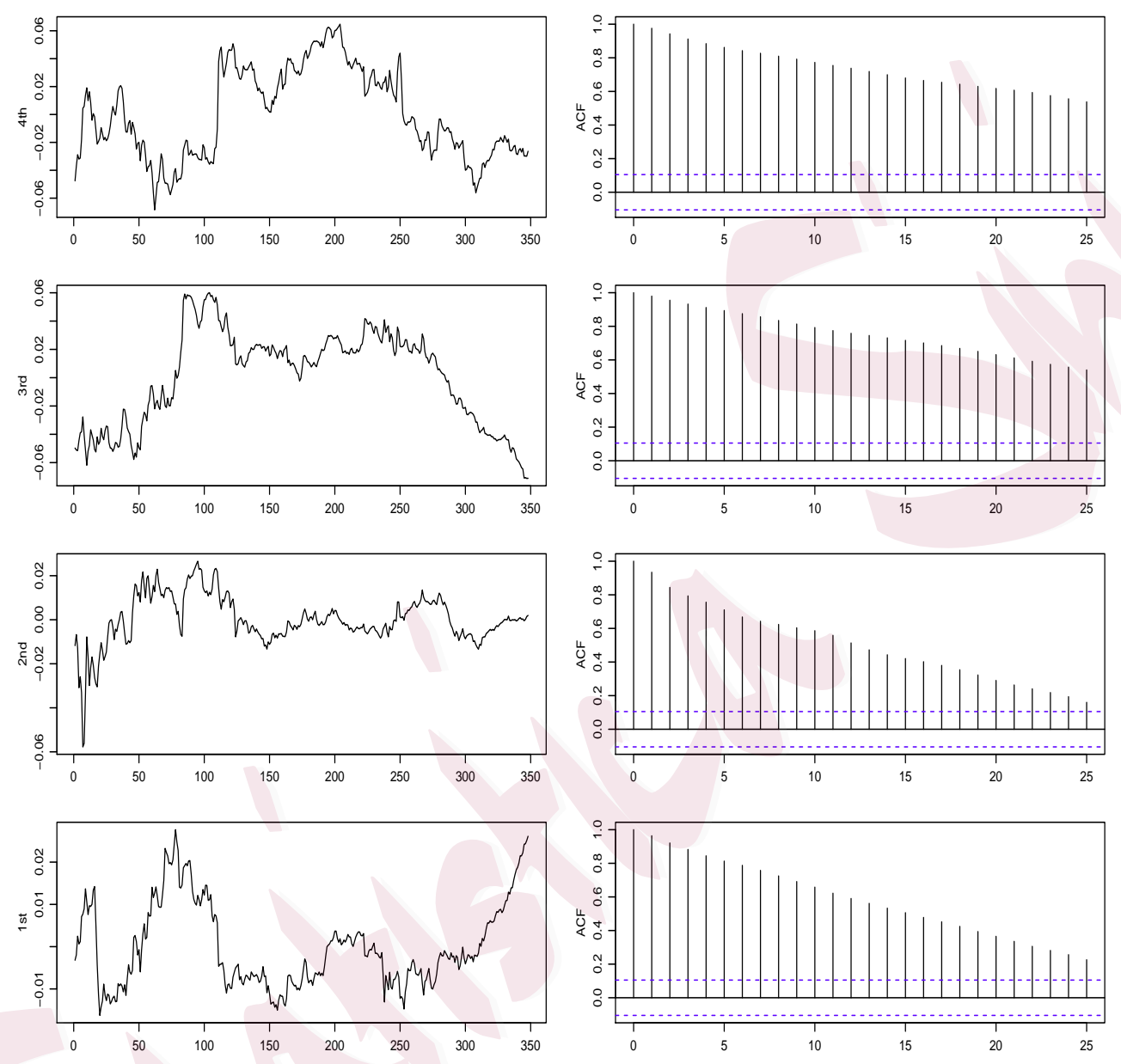

Figure 5: Time series plots of the estimated cointegrated series by the proposed method and their sample ACF for the logarithmic exchange rates based on $M=4(n / 100)^{1 / 4}$.

\section{Conclusion}

A simple, direct and model-free method for identifying cointegration relationships between high-dimensional integrated time series with possible breaks in trend is proposed in this paper. To remove the effects of the 

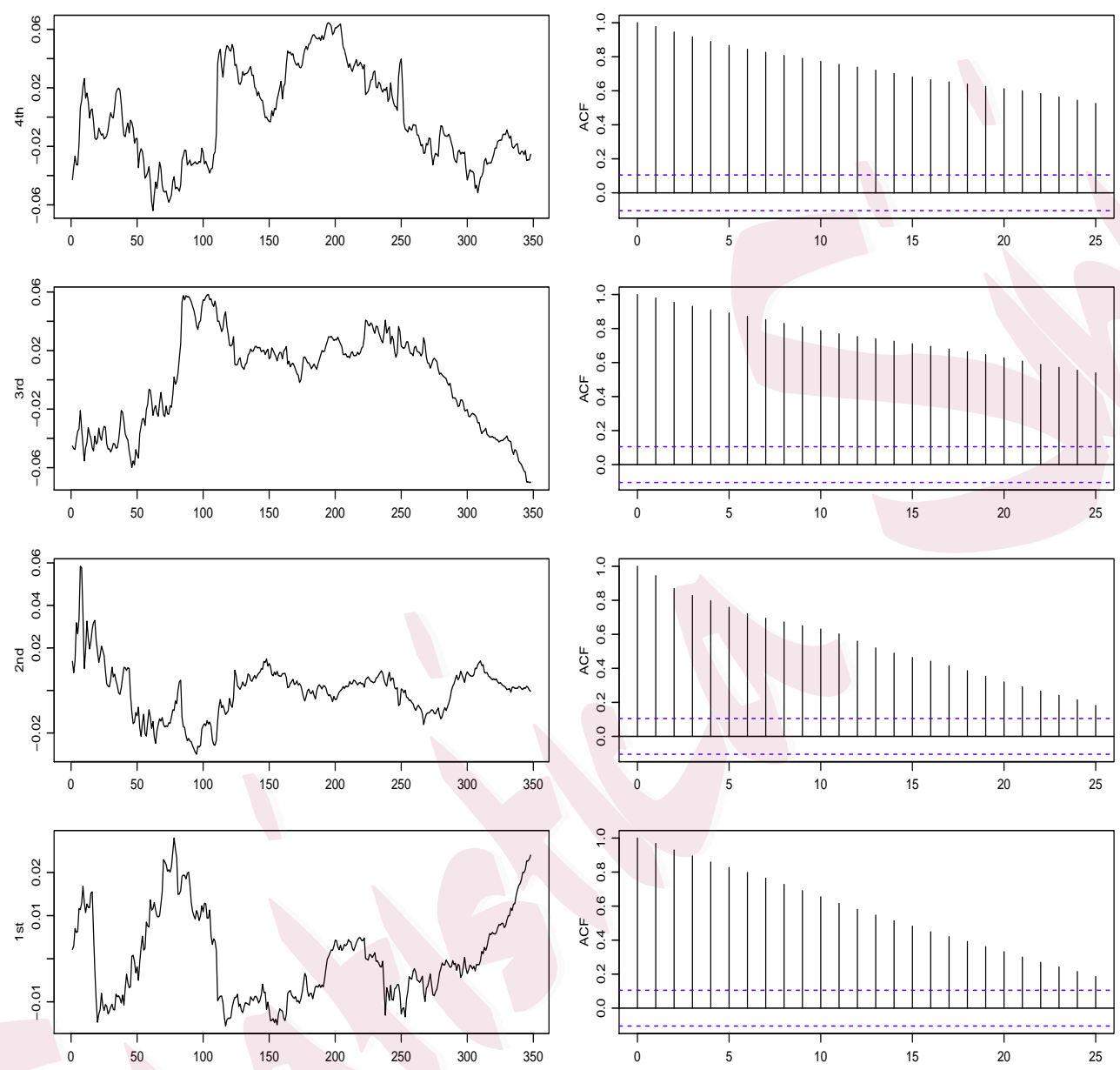

Figure 6: Time series plots of the estimated cointegrated series by the proposed method and their sample ACF for the logarithmic exchange rates based on $M=n^{1 / 4}$.

trend and the possible breaks, we propose to use the first difference of the observed series. Based on a non-negative definite matrix consisting of the Bartlett-weighted sample covariance, the cointegration space can be recovered by the eigenvectors corresponding to the smallest eigenvalues. An 
information criterion is proposed to estimate the cointegration rank, which estimates the rank consistently. In addition, unit-root tests may be applied to determine the number of stationary components of $\widehat{\mathbf{z}}_{t}$. The proposed method is different from that of ZRY, which is based on the quadratic form of the sample covariances of the observed series. Under similar conditions, the proposed procedure in this paper allows $p$ to be much larger.

This paper only focuses on inference for the cointegration rank $r$ and the cointegration space $\mathcal{M}\left(\mathbf{A}_{2}\right)$ for the case that where all the components of the cointegrated series are $I(1)$ processes and possible breaks in level. With some extra efforts, it is feasible to generalize the procedure to the case with breaks in trend. Under this circumstance, the change points can be easily detected and the proposed method can be applied to the subsamples in each regime, see Example 5 in Section 4. Further, it would be interesting to extend the procedure to cases where the integrated orders of the cointegrated series are different (see ZRY) or have fractional values (i.e., fractional cointegration (see for example Robinson and Hualde (2003), Robinson (2008) and Chen and Hurvich (2006)). These will be dealt with in future papers.

Acknowledgement We would like to thank the Co-Editor, an Associate Editor and three anonymous referees for their critical comments and thought- 
ful suggestions, which led to a much improved version of this paper. Research supported in part by grants from HKSAR-RGC-GRF No. 14325216 and the Theme-based Research Scheme of HKSAR-RGC-TBS T32-101/15R (Chan); and NSFC (No.11771390/12171427), Zhejiang provincial natural science foundation (No.LZ21A010002) and the Fundamental Research Funds for the Central Universities (Zhang).

\section{References}

Anderson, T. W. (1984). An Introduction to Multivariate Statistical Analysis (2nd Ed.). Wiley, New York.

Basu, S. and Michailidis, G. (2015). Regularized estimation in sparse high-dimensional time series models. The Annals of Statistics 43, pp. 1535-1567.

Campos, J., Ericsson, N. R. and Hendry, D. F. (1996). Cointegration tests in the presence of structural breaks. Journal of Econometrics 70, pp. 187-220.

Cavaliere, G. and Georgiev I (2007). Testing for unit roots in autoregressions with multiple level shifts, Econometric Theory, 23, 1162-1215.

Chen, W. W. and Hurvich, C. M. (2006). Semiparametric estimation of fractional cointegrating subspaces. The Annals of Statistics 34, pp. 2939-2979.

Chen, L. K. and Wu, W. B. (2019). Testing for trends in high-dimensional time series. Journal of the American Statistical Association 114, pp. 869-881.

Engel, C., Mark, N. C. and West, K. D. (2015). Factor model forecasts of exchange rates. Econometric Reviews 34, pp. 32-55.

Engle, R. and Granger, C. W. J. (1987). Cointegration and error correction: representation, estimation and testing. Econometrica 55, pp. 251-276.

Gonzalo, J., Pitarakis, J. Y. (1999). Dimensionality effect in cointegration analysis. In: Engle, R. F., White, H. (Eds.) Cointegration, Causality and Forecasting: Festschrift in Honour of Clive Granger. Oxford University Press, Oxford, pp. 212-229.

Harris, D. (1997). Principal components analysis of cointegrated time series. Econometric Theory, 13 529-557.

Harris, D., Leybourne, S. J. and Taylor, A. M. R. (2016). Tests of the co-integration rank in VAR models in the presence of a possible break in trend at an unknown point. Journal of Econometrics 192, pp. 451-467. 
Ho, M. S. and Sorensen, B. E. (1996). Finding cointegration rank in high dimensional systems using the Johansen test: An illustration using data based monte carlo simulations. The Review of Economics and Statistics 78, pp. 726-732.

Hualde, J. and Robinson, P. (2010). Semiparametric inference in multivariate fractionally cointegrated systems. Journal of Econometrics 157, pp. 492-511.

Johansen, S. (1991). Estimation and hypothesis testing of cointegration vectors in Gaussian vector autoregressive model. Econometrica 59, pp. 1551-1580.

Johansen, S. (1995). Likelihood-Based Inference in Cointegrated Vector in Gaussian Vector Autoregressive Models. Oxford University Press, Oxford.

Johansen, S. and Juselius, K. (1990). Maximum likelihood estimation and inference on Cointegration-with applications to the demand for money. Oxford Bulletin of Economics and Statistics 52, pp. 169-210.

Johansen, S., Mosconi, R. and Nielsen, B. (2000). Cointegration analysis in the presence of structural breaks in the deterministic trend. Econometric Journal 3, pp. 216-249.

Lam, C. and Yao, Q. (2012). Factor modeling for high-dimensional time series: inference for the number of factors. The Annals of Statistics 40, pp. 694-726.

Marinucci, D. and Robinson, P. (2000). Weak convergence of multivariate fractional processes. Stochastic Processes and Their Applications86, pp. 103-120.

Müller, U. K. and Watson, M. W. (2013). Low-frequency robust cointegration testing. Journal of Econometrics 174, pp. 66-81.

Newey, W. K. and West, K. (1987). A simple, positive semi-definite, heteroscedasticity and autocorrelation consistent covariance matrix. Econometrica 55, pp. 703-708.

Onatski, A. and Wang, C. (2018). Alternative asymptotics for cointegration tests in large VARs. Econometrica 86, pp. 1465-1478.

Perron, P. (1997). Further evidence of breaking trend functions in macroeconomic variables. Journal of Econometrics 80, pp. 355-385.

Phillips, P. C. B. (1991). Optimal inference for cointegrated systems. Econometrica 59, pp. 283-306.

Phillips, P. C. B. and Ouliaris, S. (1988). Testing for cointegration using principal components methods. Journal of Economic Dynamics and Control 12, pp. 205-230.

Robinson, P. M. (2008). Diagnostic testing for cointegration. Journal of Econometrics 143, pp. 206-225.

Robinson, P. M. and Hualde, J. (2003). Cointegration in fractional systems with unknown integration order. Econometrica 71, pp. 1727-1766.

Robinson, P. M. and Yajima, Y. (2002). Determination of cointegrating rank in fractional systems. Journal of Econometrics 106, pp. 217-241.

Saikkonen, P. and Lütkepohl, H. (2000). Testing for the cointegration rank of a VAR process 


\section{REFERENCES}

with structural shifts. Journal of Business \& Economic Statistics 18, pp. 451-464.

Trapani, L. (2021). Inferential theory for heterogeneity and cointegration in large panels. Journal of Econometrics 220, pp. 474-503.

Trenkler, C., Saikkonen, P. and Lütkepohl, H. (2007). Testing for the cointegrating rank of a VAR process with level shift and trend break. Journal of Time Series Analysis 29, pp. 331-358.

Zhang, R. M. and Chan, N. H. (2018). Portmanteau-type tests for unit-root and cointegration. Journal of Econometrics 207, pp. 307-324.

Zhang, R. M., Robinson, P. and Yao, Q. (2019). Identifying cointegration by eigenanalysis. Journal of the American Statistical Association 114, pp. 916-927.

Zivot, E. and Andrews, D. W. K. (1992). Further evidence on the great crash, the oil-price shock, and the unit-root hypothesis. Journal of Business \& Economic Statistics 10, pp. 251-270.

Ngai Hang Chan

The Chinese University of Hong Kong

E-mail:nhchan@sta.cuhk.edu.hk

Rongmao Zhang

Zhejiang University

E-mail: rmzhang@zju.edu.cn 